\title{
Three-dimensional position sensitivity in two-dimensionally segmented HP-Ge detectors
}

\author{
K. Vetter ${ }^{a}$, A. Kuhn ${ }^{a}$, M.A. Deleplanque, ${ }^{a}$ I.Y. Lee ${ }^{a}$, \\ F.S. Stephens ${ }^{a}$, G.J. Schmid ${ }^{b}$, D. Beckedahl ${ }^{b}$, J.J. Blair ${ }^{c}$, \\ R.M. Clark ${ }^{a}$, M. Cromaz ${ }^{a}$, R.M. Diamond ${ }^{a}$, P. Fallon ${ }^{a}$, \\ G.J. Lane ${ }^{a}$, J.E. Kammeraad ${ }^{b}$, A.O. Macchiavelli ${ }^{a}$, \\ C.E. Svensson ${ }^{a}$, \\ ${ }^{a}$ Lawrence Berkeley National Laboratory, Berkeley CA 94720, U.S.A. \\ ${ }^{b}$ Lawrence Livermore National Laboratory, Livermore CA 94550, U.S.A. \\ ${ }^{c}$ Bechtel Nevada, North Las Vegas, NV 89130-4134, U.S.A.
}

\begin{abstract}
Measured and simulated pulse shapes in electrically-segmented coaxial Ge detectors have been investigated. Three-dimensional position sensitivities have been determined experimentally and theoretically in a 36-fold segmented Ge detector. By using the two dimensional segmentation in conjunction with pulse-shape analysis, a position sensitivity of better than $1 \mathrm{~mm}$ can be obtained in three dimensions at an energy of $374 \mathrm{keV}$. This is achieved by analyzing the shape of net charge signals of segments containing interactions and of transient image charge signals of neighboring segments. The ability to locate interactions in three-dimensions is one of the crucial properties in the proposed $\gamma$-ray energy tracking array (GRETA). The concept of $\gamma$-ray tracking will not only increase the efficiency in detecting $\gamma$ radiation but also enables the localization and characterization of unknown $\gamma$-ray sources with much higher accuracy than is possible with current instruments.
\end{abstract}

PACS: 07.85; 29.40; 29.40.K; 29.40.W

Key words: HPGe detectors; position-sensitive detectors; GRETA; gamma-ray tracking 


\section{Introduction}

Currently, a new technique to enable the tracking of $\gamma$ radiation is being developed which will allow the determination of the time sequence of interactions of $\gamma$ rays and their respective positions in a detector as well as their energies [1]. An implementation of this concept, the gamma-ray energy tracking array, known as GRETA, is currently being developed at the Lawrence Berkeley National Laboratory (LBNL) and focuses on improving the performance of $\gamma$-ray spectrometers primarily in the field of nuclear structure physics. It is anticipated that GRETA will improve the resolving power over existing arrays, such as Gammasphere, by at least two orders of magnitude [1,2]. This large gain is based on an increased efficiency and peak-to-total ratio, a better Doppler-shift correction, and a higher count-rate capability. The concept of $\gamma$-ray tracking also allows potential improvements in the ability to locate and characterize unknown $\gamma$-ray sources, useful in areas such as astrophysics or medical $\gamma$-ray imaging.

One crucial ingredient in the concept of gamma-ray tracking is the ability to determine the energy and position of individual interactions, which requires a three-dimensional position resolution on the order of a few millimeters. In the approach we are pursuing, the determination of the position is achieved by employing pulse-shape analysis in a two-dimensionally segmented coaxial HP-Ge detector. While pulse-shape information is already being used successfully in Ge detectors to improve energy resolution [3,4], to perform Compton suppression [5,6], and to determine the mean radius of interactions for an improved Doppler correction [7], it is clearly not fully explored. For example, in [7] a mean radius of all interactions of one $\gamma$ ray is obtained by only considering one or two parameters of the signal. These studies have recognized already that the pulse shape reflects energies as well as positions of interactions of the $\gamma$ ray with the detector material. However, without segmentation the pulse shape is only sensitive to one dimension, the direction of the charge drift, e.g. the radius in coaxial or the depth in planar detectors. To overcome this limitation, we are using a closed-ended coaxial Ge detector with its outer contact divided into two-dimensional segments. With sufficiently small segments, charge is not only induced on the charge-collecting segment but also transiently on the neighboring segments. Since the segmentation is perpendicular to the electric field lines (e.g. the radius) a position sensitivity in the complementary two dimensions (e.g. the depth and azimuthal angle) can be obtained. In this paper we report on measurements and calculations of the position sensitivity of the GRETA prototype detector. 


\section{GRETA prototype detector}

The measurements to determine the three-dimensional position sensitivity in segmented coaxial Ge detectors have been performed with a GRETA prototype detector delivered by Eurisys Measures in Strasbourg. This prototype is illustrated in fig. 1. It consists of a closed-ended HP-Ge n-type crystal with a tapered hexagonal shape designed to fit in a spherical shell arrangement consisting of about 120 of these detectors. The length of the crystal is $9 \mathrm{~cm}$, the diameter at the back is $7 \mathrm{~cm}$ and the maximum diameter at the front is $4.4 \mathrm{~cm}$. The angle of the taper is 10 degree. The outer electrode is divided into 36 segments, 6 longitudinal and 6 transverse. The 6 longitudinal boundaries are located in the middle of the flat sides of the hexagonal shape. The width of the transverse segments starting at the front (the narrow side) are $7.5 \mathrm{~mm}$, $7.5 \mathrm{~mm}, 15 \mathrm{~mm}, 20 \mathrm{~mm}, 25 \mathrm{~mm}$ and $15 \mathrm{~mm}$, respectively. The thicknesses of the layers were chosen to distribute the number of the interactions more equally among the segments for $\gamma$ rays coming from the front and to allow the study of the influence of different thicknesses on the transient-signal sensitivity. The Ge crystal resides in a $1 \mathrm{~mm}$ thick aluminum can of the same shape as the crystal. This can is separated from the crystal by $1 \mathrm{~mm}$ to simulate a close packing of individually encapsulated detectors, similar to the design of the Cluster detectors [8] currently implemented in the Euroball array. The 37 FETs for the 36 segments and the central channel are located and cooled in the same vacuum as the crystal. Cold FETs provide low noise which is important for optimizing the energy and position resolution. The charge sensitive preamplifiers are positioned on a cylindrical motherboard next to the vacuum feedthroughs in the back of the detector. These preamplifiers and their mount were designed and built at LBNL and are characterized by their small size, fast risetime, low noise, and excellent response properties [9]. More details on the GRETA prototype detector and the preamplifiers can be found in [10].

\section{Origin and calculation of charge signals in a segmented Ge de- tector}

To determine the position in three dimensions based on pulse-shape analysis, a detailed understanding of the pulse shapes is necessary. In the detector, a signal is produced when electrons and holes, formed by the slowing down of the photo- or Compton-electrons, induce an image charge of opposite sign on the electrodes. As the charge drifts toward the electrodes, the amount of the image charge changes and currents flow into or out of the electrodes. When the charge is at a large distance from the electrodes, the induced charge is distributed over several electrodes. As the charge moves closer to the desti-

nation electrode, the induced charge on this electrode increases and charges 
on the other electrodes decrease until the charge finally reaches the electrode and neutralizes the image charge. The observation of a net charge on the destination or charge-collecting electrode can be used to identify the electrode which contains the interaction. The predominant characteristic of the transient image signals is that they vanish when the charge carriers are collected and that either polarity is possible, dependent on the different contributions of holes and electrons. The fact that either polarity is possible is useful since it increases the dynamic range of signals, increasing the position sensitivity. For instance, simply observing the polarity of the induced signal allows to distinguish between an interaction at small and large radius. However, the drawback of the two polarities is that the contributions of holes and electrons can compensate each other, generally close to the mid point between the two electrodes, which results in a reduced sensitivity at these locations.

Net charge, as well as transient image charge signals can be calculated in the following manner: first we have to calculate the path of the charge carrier for a given position of the interaction. The motion of the charge carriers is determined by the electric field $\vec{E}(\vec{r})$, which itself depends on the detector geometry, applied voltage $\mathrm{V}_{0}$, and intrinsic space charge density $\rho$ and mobility $\mu$. To obtain the electric field the Poisson equation

$$
\Delta \Phi(\vec{r})=-\rho(z) / \epsilon
$$

for the potential $\Phi$ is solved. Here, $\epsilon$ is the dielectric constant for Ge and $\rho(\mathrm{z})$ reflects the dependence of the space charge density on the depth. We used a linear variation for the impurity density from $-11 \times 10^{-9} \mathrm{~cm}^{-3}$ at the front to $-6 \times 10^{-9} \mathrm{~cm}^{-3}$ at the back of the detector, as suggested by the manufacturer. After solving the Poisson equation numerically employing either the relaxation method or finite element methods, the potential is obtained on a three dimensional grid with a grid size of e.g. $1 \mathrm{~mm}$. The calculated potential for the tapered hexagon geometry at a depth of $4 \mathrm{~cm}$ is shown on the left side of fig. 2. The electric field is then calculated on these grid points as

$$
\vec{E}(\vec{r})=-\nabla \Phi(\vec{r})
$$

Using the relation

$$
\vec{v}(\vec{E}(\vec{r}))=\mu(T, E(r), \zeta, \vartheta) \vec{E}(\vec{r})
$$

for the velocity, we can calculate the trajectory for electrons and holes for any given start position by interpolating the electric field between the grid points and a given time interval $\Delta \mathrm{t}$ of (e.g.) $2 \mathrm{~ns}$, small enough to prevent discontinuities in the drift velocity. We have to point out that the mobility is not only a function of the temperature and electric field but depends also 
on the angle $\zeta$ between the drift direction and the crystal orientation and the angle $\vartheta$ between the electric field and the crystal orientation. The temperature, electric field strength and crystal orientation dependence for the magnitude of the mobility for electrons and holes in germanium can be taken from [11] and [12], respectively. The effect of a velocity vector which is not parallel to the electric field direction due to the anisotropic effective mass tensor in Ge [13] is discussed in [14]. We assume that the slowing down time of the original Compton electrons, with the related creation of electron-hole pairs, as well as the time for these pairs to reach the velocity $v(\vec{E}(\vec{r}))$ is negligible. Furthermore, we neglect the range of the Compton electron and the finite size of the distribution of charge carriers moving towards the electrodes. The most critical assumption is to neglect the range of the Compton electrons and will be discussed later in more detail. Fig. 3 shows calculated trajectories for electrons and holes assuming an interaction took place at $\mathrm{x}=2.2 \mathrm{~cm}, \mathrm{y}=0.45 \mathrm{~cm}$ and $\mathrm{z}=5.85 \mathrm{~cm}$ in a coordinate system indicated in the figure.

To finally calculate the induced signals in the different segments we use Ramo's theorem for the so called weighted potential [15]. This potential is derived by solving the Laplace equation in the given geometry with voltage only on the sensing electrode and all the other contacts on ground. The right-hand side of fig. 2 shows the weighted potential for one of the segments at the fourth layer. Calculating the electric field for every grid point and every segment as before and using the previously obtained trajectories for holes and electron we can determine the induced charge $\Delta Q_{i j}$ (i:holes or electron, $\mathrm{j}$ :number of segment) of a charge $q_{0}$ for each step $\Delta \overrightarrow{r_{i}}$ :

$$
\Delta Q_{i j}=q_{0} \vec{E}_{j}\left(\overrightarrow{r_{i}}\right) \Delta \overrightarrow{r_{i}} / V_{0}
$$

In the following, $q_{0}$, which reflects the energy deposit per interaction, is normalized to 1 or $100 . \mathrm{V}_{0}$ is the applied voltage, in our case $+3000 \mathrm{~V}$. The lower part of fig. 3 shows calculated signals for the indicated starting point of the trajectory in segment B4. The left hand side shows the net charge signal of segment B4 and the different contributions of holes and electrons. The right hand side shows transient charge signals of the azimuthal neighbor $\mathrm{C} 4$ and the segment in front B3.

\section{Measurement of signals}

To confirm the accuracy of the above described calculations it is necessary to compare the resulting signals with measured pulse shapes. In addition, the measurements will determine the position sensitivity which is achievable. An experiment was assembled to enable the measurement of signals for 9 Ge segments for a given single interaction point in the volume of the detector. This is 
realized by using a collimated ${ }^{137} \mathrm{Cs} \gamma$-ray source placed in front of the GRETA prototype detector and requiring a coincidence between this detector and one of three $\mathrm{NaI}$ detectors which are located at 90 degree at an adjustable depth. The setup is shown in fig. 4. While the collimator in front of the Ge detector defines the $\mathrm{x}$ and $\mathrm{y}$ position of the interactions, the slit in the collimator in front of the NaI detectors defines the depth (z) of the interactions. The NaI detectors were chosen instead of Ge detectors because of their size which allows a large solid angle coverage in a close geometry. All three NaI counters measure 5" in diameter and 6" in length. To define the location of the interactions as precisely as possible, the diameter of the hevimet collimator hole in front of the Ge detector as well as the slit width of the lead absorbers in front of the NaI detectors were only $1 \mathrm{~mm}$. To select events with only one interaction, gates on the energies deposited in the Ge and the NaI detectors were used, implying a Compton scattering process of 90 degree, which deposits $374 \mathrm{keV}$ in the Ge crystal. This improvement is indicated in fig. 5 which shows result of Monte-Carlo simulations performed with GEANT-3 [16]. The top part shows the number of interactions at the given position with and without an energy gate in both detector systems. Without the gate only about $55 \%$ of the coincidence events are due to single interactions compared to more than $85 \%$ with the energy requirement. In the lower part of this figure the spread in the positions of the interactions based on the $1 \mathrm{~mm}$ collimation is shown. The size of the cylindrical volume which has been mapped out has a diameter of about $1.7 \mathrm{~mm}$ and a depth of about $1.9 \mathrm{~mm}$, both measured at the full width half maximum. Using the $1 \mathrm{~mm}$ collimation system and a ${ }^{137} \mathrm{Cs}$ source with an activity of $1 \mathrm{mCi}$ we obtain a count rate of about 1 event in 10 to 60 minutes depending on the radial position of the front collimator at a depth of $4 \mathrm{~cm}$ in the Ge. The ${ }^{137} \mathrm{Cs}$ source was specially built to have all the activity in a cylinder with a diameter of $1 \mathrm{~mm}$. Comparing with a source of higher $\gamma$-ray energy (e.g. ${ }^{60} \mathrm{Co}$ ), the advantage of the lower $\gamma$-ray energy of ${ }^{137} \mathrm{Cs}$ is that the collimators can be shorter providing a higher count rate.

The signals of 9 segments which are arranged in a $3 \times 3$ matrix were taken from the preamplifier into fast amplifiers to match the input range of the waveform digitizers. In addition, the preamplifier signals of the three NaI detectors were also amplified and fed into the waveform digitizer system. Each of the 12 channels of the waveform digitizer has a $500 \mathrm{MHz}$ sampling rate and a pulse height resolution of 8 bits. The collimation system was arranged to allow the central segment (B4) of the $3 \times 3$ matrix of segments to contain the interaction resulting in a net charge signal while the 8 neighbor segments observe only the transient image charge signals. Measured sets of signals of the 9 segments are shown in fig. 6 and fig. 7 at different radii of the interaction point. The numbering of the segments is the same as in fig. 3. For comparison, calculated signals at the given positions are plotted as dashed lines. While the agreement between calculations and measurements at the large radius is very good, the calculated signal at the smaller radius is faster than the measured one indicating a too fast hole mobility. To improve the signal-to-noise ratio 
in the measured signals, we averaged 16 samples at $500 \mathrm{MHz}$ to give a $32 \mathrm{MHz}$ sampling rate which is close to the bandwidth of the prototype detector and preamplifier system [10].

The alignment of the detector relative to the scanning and collimation system was done by scanning the front face with a collimated source and plotting the intensity of the $662 \mathrm{keV}$ transition in ${ }^{137} \mathrm{Cs}$ measured for a fixed time for all segments as a function of the position. By identifying the segment boundaries as a function of depth and azimuthal angle the alignment could be determined and adjusted. The location of the detector segments in the $\mathrm{z}$ (depth) direction were obtained by scanning a collimated ${ }^{241} \mathrm{Am}$ source from the front to the back of the detector and measuring the intensity of the $60 \mathrm{keV} \gamma$-ray as a function of depth for each segment.

So far, we have measured 36 different positions which required about 6 weeks of continuous data taking. These 36 positions are composed of 12 measurements for each of three different depth $(\mathrm{z})$ layers. The $\mathrm{x}-\mathrm{y}$ locations are indicated in fig. 8. The distribution of interaction positions were derived by simulations. In the $\mathrm{x}$ direction, which is along a transversal segmentation line, we moved in $4 \mathrm{~mm}$ steps from $22 \mathrm{~mm}$ to $10 \mathrm{~mm}$. In the y direction perpendicular to the $\mathrm{B} / \mathrm{C}$ boundary we moved in $3 \mathrm{~mm}$ steps, starting at a distance of $1.5 \mathrm{~mm}$ from $\mathrm{C} 4$. In the same way, we moved in the $\mathrm{z}$ direction in $3 \mathrm{~mm}$ steps starting at distance of $1.5 \mathrm{~mm}$ from neighbor B3 in B4.

\section{Position sensitivity}

Using the measured signal shapes from the 36 positions it is possible to obtain a position sensitivity based on signal-shape differences in this segmented Ge detector. The sensitivity we refer to in the following quantifies the change of signal shapes as a function of the location of the interactions relative to the observed noise which represents the main uncertainty in the measurement. We assume that the distance between the positions we measure is small enough to allow to neglect e.g. crystal orientation effects and the uncertainty in the overall alignment of the detector relative to the collimation system of the order of about $1 \mathrm{~mm}$. The agreement between the experimental results and the calculations seem to justify this approach with its inherent simplifications.

An indication of the obtainable position sensitivity can already be seen in the apparent spread of the signals in fig. 6 and in fig. 7. To determine the contribution of the finite opening angle of the collimation system to this spread we performed Monte-Carlo simulations using GEANT with the set-up drawn in fig. 4. Energies and positions of each interaction from these simulations were used to derive calculate signals in the 9 segments studied in the experiment. To compare the spread in the signals obtained from measurement and the calculation, we plot the distributions of maximum amplitudes of the transient 
image charge signals for different positions and segments. In fig.9 distributions of measured and calculated amplitudes are shown for signals in segment $\mathrm{C} 4$ for different distances from the B/C boundary (y) and constant values of $\mathrm{x}$ and $\mathrm{z}$. While these distributions indicate the sensitivity in the azimuthal direction, fig. 10 illustrates the sensitivity in the depth (z) by showing maximum transient charge amplitudes in segment B3 for different z-positions and constant $\mathrm{x}$ - and $\mathrm{y}$-values. Besides the good agreement between the measured and calculated distributions, the dependence of the maximum induced charge on the location of the interaction relative to the two different boundaries can be clearly seen. To illustrate the sensitivity for the third dimension ( $\mathrm{x}$, which is similar to the radius) contained in this one parameter we show the transient charge amplitudes in segment B3 again but now for different $\mathrm{x}$-values in fig. 11. The positions and the width of the distributions are more spread out than in the previous two figures, indicating a higher sensitivity. The smaller number of events for smaller x-values reflects the increase of the absorption probability of the $\gamma$ rays after the 90 degree scattering.

To further analyze the comparison we plot the mean as well as the width of these distributions as a function of position as shown in fig. 12, again for segments C3, C4 and B3 for the three different depth values, $\Delta \mathrm{z}$, x-values of $22 \mathrm{~mm}$ and $14 \mathrm{~mm}$ and all available y-values. While the width of the peaks can be reproduced very well, the location of the mean values show some systematic deviations. These discrepancies are likely to reflect the uncertainty in the overall alignment of the prototype detector relative to the collimation system and crystal orientation effects which have not yet been taken into account completely. The good agreement, especially of the width of the distributions, indicates that the major cause for the observed spread is only due to the finite size of the collimation system and not due to a limited sensitivity e.g. the noise in the signals. The larger spread in the mean values as well as the larger width of the distributions closer to the boundaries imply a higher sensitivity at these locations.

To quantify the differences in the signals between all combinations of pairs of locations we will in the following take the entire signals, e.g. the time samples between the $10 \%$ (t10) and the $90 \%$ (t90) level of the net charge signal into account. Comparing all pairs of interaction points ensures that each set of signals is unique in the volume studied. The difference in the signal amplitudes $q^{k}(t)$ at the time sample $t$ between the positions $i$ and $j$ has to be related to the uncertainty in the signals, the noise level $\sigma^{k}$, which is measured in each of the segments $\mathrm{k}$, and the distance of the positions $\Delta r_{i j}$. Since we compare two signals of the same segment with noise level $\sigma^{k}$, the total noise contribution is $\sqrt{2} \times \sigma^{k}$, assuming the noise is not depending on the position of the interaction. Thus, the sensitivity $S_{i j}$, e.g. the ability to distinguish two locations $i$ and $j$ of interactions based on signals measured in 9 segments, can be defined 
in the following way:

$$
S_{i j}^{2}=\frac{\Delta r_{i j}^{2}}{\Delta s_{i j}^{2}}
$$

where $\Delta s_{i j}$ quantifies the difference of the signals in terms of the measured noise:

$$
\Delta s_{i j}^{2}=\sum_{k=1}^{9} \sum_{t=t 10}^{t 90}\left[\frac{\bar{q}_{i}^{k}(t)-\bar{q}_{j}^{k}(t)}{\sqrt{2} \sigma^{k}}\right]^{2} .
$$

Therefore, a high sensitivity refers to a small value of $S_{i j}$. If the set of signals differ only by $\sigma_{i j}$ for two locations $i$ and $j$, the sensitivity will be just $\Delta r_{i j}^{2}$. Several remarks seem appropriate at this point concerning the details of the described procedure: First of all, as $\bar{q}_{i}^{k}(t)$ indicates, signals are used which are averaged over many measurements for each segment and position. The main reason for this is to generate one set of signals which corresponds to the center (average) position of the collimation system. As indicated in the measured signals above, the spread in the signals even for a volume of $\approx 3 \mathrm{~mm}^{3}$ is significant, preventing the determination of a signal at a fixed position on an event-by-event basis. Another goal achieved by the averaging is the removal of noise, thus leaving a signal which reflects purely the position variation. The noise level $\sigma^{k}$ was determined by extracting the standard deviation of 500 time samples of one of the individual events, excluding the signal region. The average noise level $(1 \sigma)$ for all the 9 channels considered was about $5 \mathrm{keV}$. This value agrees with a more sophisticated approach in which the total noise as a function of frequency is measured. At a frequency of about $40 \mathrm{MHz}$, which is the bandwidth of the different channels, we obtain also a value of about $5 \mathrm{keV}$ [10]. Fig. 13 and fig. 14 show averaged signals for the indicated positions for both the measurements and the calculations. Also included are the calculated signals for a single interaction at the targeted position. At the outside radii the assumption of the average signal representing the center position appears well justified while for smaller radii the agreement is not as good. This is due to the low statistics of only 10-20 counts and the high position sensitivity at these locations and the discrepancy in the mobility of the holes as mentioned earlier.

Two examples of experimentally obtained sensitivities are shown in fig. 15 and fig. 16. These graphs display sensitivities for positions indicated by the arrows relative to all other 35 positions. For instance, in fig. 15, values of $S_{i j}$ are plotted where the position $i$ is at $\mathrm{x}=22 \mathrm{~mm}, \mathrm{y}=1.5 \mathrm{~mm}$ and $\Delta \mathrm{z}=4.5 \mathrm{~mm}$ while $j$ represents all other positions of the collimation system. Again, the three columns display the three different z-layers at a distance of $1.5 \mathrm{~mm}$, $4.5 \mathrm{~mm}$ and $7.5 \mathrm{~mm}$ away from the front segment, respectively, each containing 12 location of interactions. While the magnitudes in fig. 15 represent 
roughly an average of all combinations, the position in fig. 16 is located in a place with lowest sensitivity, where electrons and holes compensate each other leading to small transient induced signals which results in a low sensitivity in directions perpendicular to the azimuthal boundaries. However, even for these worse cases a sensitivity of $\lesssim 1 \mathrm{~mm}$ is obtained for an energy deposition of $374 \mathrm{keV}$. For other locations or radial directions the obtainable sensitivities are better than $0.5 \mathrm{~mm}$. For comparison, results employing the same procedure for calculated signals are displayed in the middle row. While the signals and the differences were obtained purely by calculations, the noise were taken from the experimental data the same way as above. The lower parts of these figures show the calculated sensitivity assuming the interaction takes place right on the center of the collimation system. Generally, a very good agreement between the measured and the calculated sensitivities is achieved. The obtained calculated values show very similar dependencies of positions as the measured ones and also the magnitude agrees very well.

It is interesting to study the direction dependence of the derived sensitivities in more detail. As can already be seen from fig. 15 and fig. 16, the x-direction has usually a higher sensitivity than the y- or z-direction. This is due to the larger variation in the shape for changing radii than for the other directions. Since the underlying mechanism of the transient induced signal along the $\mathrm{y}$ and $\mathrm{z}$ direction is the same, the sensitivity along these directions is comparable. On average, a sensitivity of about $0.2 \mathrm{~mm}$ can be deduced for the radial direction and about $0.5 \mathrm{~mm}$ for the two directions perpendicular to the boundaries. In fig. 17 the sensitivity from all position pairs $(36 \times 36)$ are summarized for an energy deposition of $374 \mathrm{keV}$. Not only all locations appear to be unique but most of the positions show a sensitivity of better than $0.5 \mathrm{~mm}$. This remarkable result indicates that the size of the segments is adequate and the noise level is low. Table 1 shows the derived sensitivities as a function of depth. These numbers represent averages over all combination for each layer. Included are also the numbers obtained by the two types of calculations. The degradation in sensitivity for larger values for $\Delta \mathrm{z}$ reflects the saturation effect of the transient signals for larger distances to boundaries which could be already seen in previous figures, showing the distributions of maximum induced amplitudes. The sensitivity in the x-direction of about $2 \mathrm{~mm}$ at an energy of $374 \mathrm{keV}$ obtained here is in good agreement with results of a recent study on the achievable radial resolution possible in principle in coaxial Ge detectors [17].

Finally, we can also study the dependence of energy on the sensitivity by scaling the noise amplitude accordingly. At low energy the signal-to-noise ratio is worse. However, we still obtain a position sensitivity of about $1 \mathrm{~mm}$ at an energy of $100 \mathrm{keV}$. 


\section{$6 \quad$ Sensitivity and $\gamma$-ray tracking}

In this section we discuss how the excellent position sensitivity obtained above relates to the performance of a $\gamma$-ray tracking detector.

The sensitivity we refer to in this report represents a position resolution neglecting effects such as the range of the Compton electrons or the broadening of the distribution of charge carrier traveling towards the electrodes due to diffusion and assuming a single interaction without further complications of disentangling multiple interactions. In particular the range of the Compton electron represents an uncertainty which will limit the accuracy in determining the location of the scattering process. For instance, in the current measurements, the Compton electron of $374 \mathrm{keV}$ has a range of about $0.2 \mathrm{~mm}$ which is of the same order as the sensitivity obtained for many positions. The only reason we were able to achieve a sensitivity sometimes even better than the range of the Compton electron is due to the averaging procedure and using a slit in front of the NaI detectors to define the 90 degree. scattering. For a Compton electron of $1 \mathrm{MeV}$ (e.g. 90 degree Compton scattering of a $1.4 \mathrm{MeV}$ $\gamma$ ray) the range increases to about $1 \mathrm{~mm}$ [18]. The maximum diameter of the distribution of charge carriers can be estimated to be less than $0.1 \mathrm{~mm}$ in the present GRETA prototype geometry and therefore does not represent a serious limitation.

While in this paper we discussed only the sensitivity for localizing one interaction in the volume of a Ge detector, this is only a necessary first step for determining the locations of multiple interactions occurring in the same or neighboring segments. In such cases the position resolution will have additional contributions from the algorithm used to disentangle these multiple interactions.

Another effect which has no impact on the position determination of a single interaction, but does for the proposed $\gamma$-ray tracking based on events with at least two interactions, is the Compton profile. It reflects the initial momentum distribution of the electrons involved in the Compton scattering. Generally, in the description of Compton scattering the binding energy as well as the momentum of the electron is neglected. However, the momentum of the electron has a measurable effect on the angle-energy relation for $\gamma$-ray energies up to several $\mathrm{MeV}$, and must be considered if the accuracy in position determination is required to be of the order of $1 \mathrm{~mm}$. In contrast to the range of the Compton electron, this effect becomes more important for smaller $\gamma$-ray energies and also depends on the scattering angle. Evidence for the importance of this effect is shown in fig. 18 which displays measured and calculated energy spectra obtained in the GRETA detector mounted in the collimation system with a hole diameter as well as slit size of $1 \mathrm{~mm}$ as above. Here, the momentum distribution of the electrons in Ge [19] has to be taken into account to obtain an agreement between the measured and the calculated energy profile in the Ge detector. 
Another effect which could limit the accuracy in the determination of the location of interactions is the anisotropy of the charge carrier velocity with respect to the crystal orientation. Both the magnitude of the drift velocity and the angle between the velocity and electric field changes relative to the crystal orientation. The magnitude has a variation of $0-15 \%$ and the the angle variation is about $0-15$ degree at a electric field strength of $1 \mathrm{KV} / \mathrm{cm}$. Both effects correspond to an uncertainty of up to a few millimeters [14].

\section{Conclusions}

We have performed measurements and calculations to study the three-dimensional position sensitivities for single interactions of $\gamma$ rays in segmented Ge detectors. The position sensitivity was achieved by applying digital pulse-shape analysis to the induced signals from a two-dimensionally segmented detector. We used the 36-fold segmented GRETA prototype detector in a coincidence set-up which allowed us to localize single interactions in the crystal. Pulse-shape calculations in combination with Monte-Carlo simulations of $\gamma$-ray interactions have been performed which yield good agreement with the measured signals and their properties for the different positions. A position sensitivity of better than $0.5 \mathrm{~mm}$ has been obtained for most positions in the measured range of the crystal. The crystal volume studied includes most of the radial extension of the crystal and up to $1 \mathrm{~cm}$ away from segment boundaries. Furthermore, we found that the sensitivity in the radial direction of about $0.2 \mathrm{~mm}$ is higher than for the directions perpendicular to the segmentation lines where we obtain about $0.5 \mathrm{~mm}$. By studying the energy dependence of the position sensitivity we found that even for a $\gamma$-ray energy of $100 \mathrm{keV}$ a sensitivity of about $1 \mathrm{~mm}$ can be achieved. The excellent position sensitivity which has been demonstrated in three dimensions makes the concept of $\gamma$-ray tracking feasible.

\section{Acknowledgements}

We would like to thank Michael Maier and Harold Yaver for the design, construction and implementation of the superb preamplifiers and Eurisys Measures for providing an unprecedented detector. We also thank Bill Payne for supplying the data acquisition program. This work was supported by the Director, Office of Energy Research, Division of Nuclear Physics of the Office of High Energy and Nuclear Physics of the U.S. Department of Energy under contract No. DE-AC03-76SF0093. 


\section{References}

[1] M.A. Deleplanque, et al., Nucl. Instr. and Meth. A 430 (1999) 292

[2] G.J. Schmid, et al., Nucl. Instr. and Meth. A 430 (1999) 69

[3] W. Gast, et al., Nucl. Instr. and Meth. A 171 (1980) 49

[4] B. Hubbard-Nelson, et al., Nucl. Instr. and Meth. A 422 (1999) 411

[5] B. Philour, et al., Nucl. Instr. and Meth. A 403 (1998) 136

[6] G.J. Schmid, et al., Nucl. Instr. and Meth. A 422 (1999) 368

[7] Th. Kröll, et al., Nucl. Instr. and Meth. A 371 (1996) 489

[8] J. Eberth, et al. Nucl. Instr. and Meth. A 369 (1996) 135

[9] M.R. Maier, et al., submitted to the Proceedings of the IEEE Symposium on Nuclear Science, Seattle Oct. 1999

[10] K. Vetter, et al., submitted to Nucl. Instr. and Meth. A

[11] G. Ottaviani, C. Canali and A. Alberigi Quaranta, IEEE Trans. Nucl. Sci. NS$22(1975) 192$

[12] L. Reggiani, C. Canali, F. Nava, and G. Ottaviani, Phys. Rev. B 16 (1977) 2781

[13] W. Sasazaki, and M. Shibuya, J. Phys. Soc. Japan 11 (1956) 1202

[14] K. Vetter, et al., in preparation for publication in Nucl. Instr. and Meth. A

[15] S. Ramo, Proc. IRE (1939) 584

[16] GEANT3, Detector Description and Simulation Tool (CERN, Geneva, 1993)

[17] J. Blair, et al., Nucl. Instr. and Meth. A 422 (1999) 331

[18] T. Mukoyama, Nucl. Instr. and Meth. A 134 (1976) 125

[19] F. Briggs, et al., Atomic Data Nuclear Data Tables 16 (1975) 201 
Table 1

Sensitivities for each of the three studied layers in $\mathrm{mm}$. The values represent average sensitivities for all combinations per layer.

\begin{tabular}{|l|c|c|c|}
\hline$\Delta \mathrm{z}[\mathrm{mm}]$ & 1.5 & 4.5 & 7.5 \\
\hline Measurement (averaged signals) & 0.33 & 0.34 & 0.39 \\
\hline Simulation (averaged signals) & 0.29 & 0.31 & 0.38 \\
\hline Simulation (single interaction) & 0.22 & 0.25 & 0.28 \\
\hline
\end{tabular}




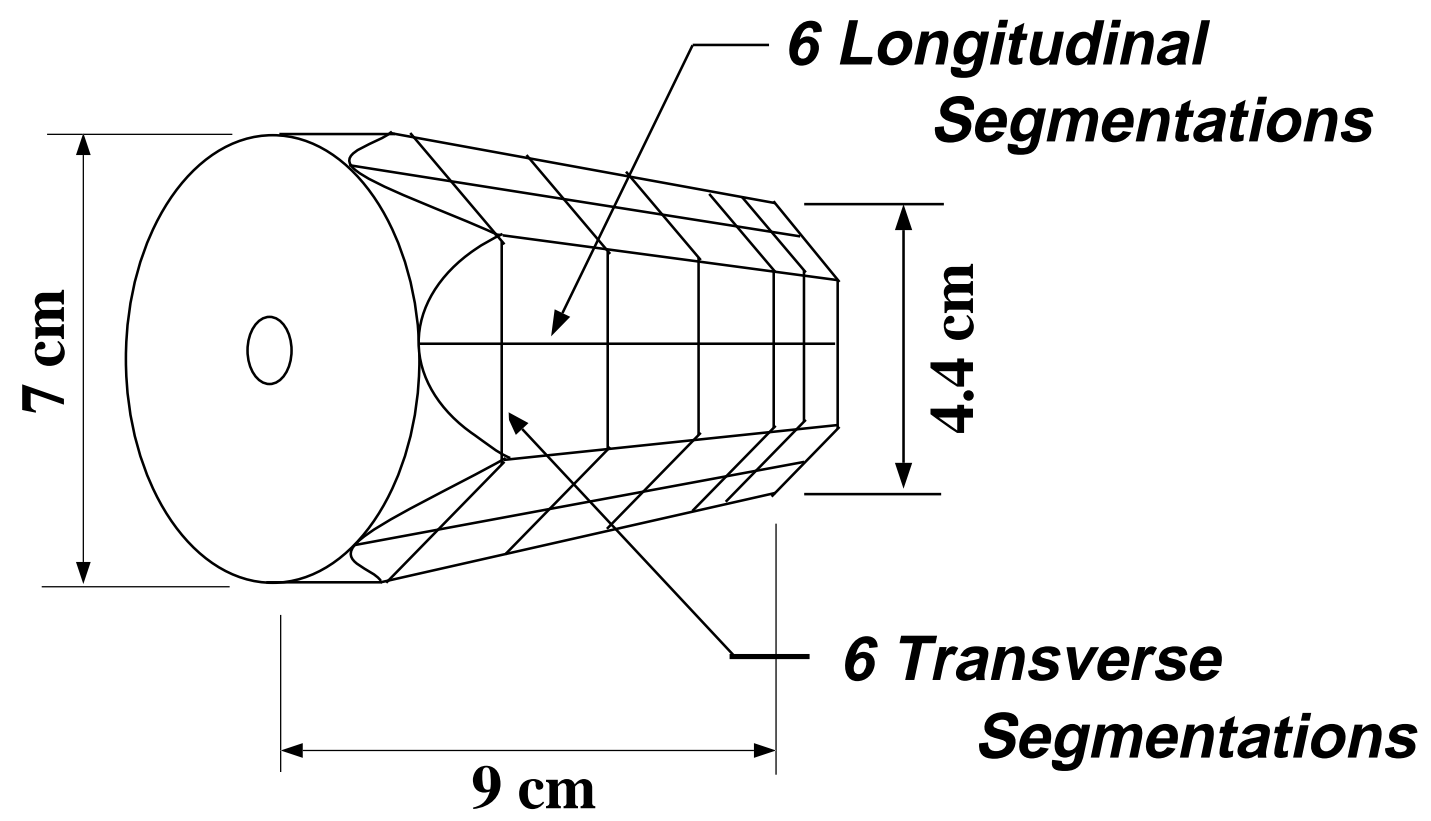

Fig. 1. The GRETA prototype detector with its tapered hexagonal shape and the arrangement of the 36 segments. 

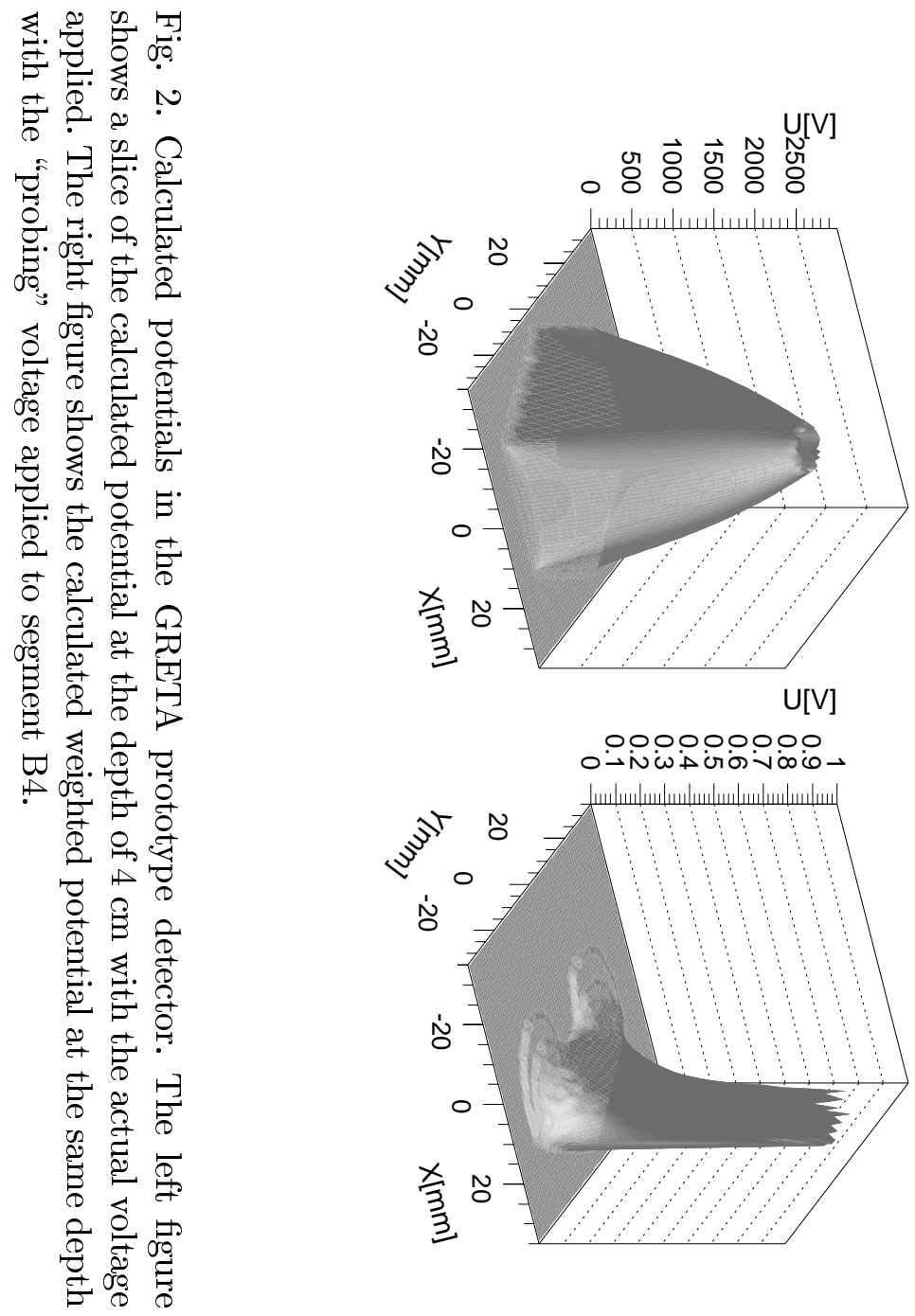

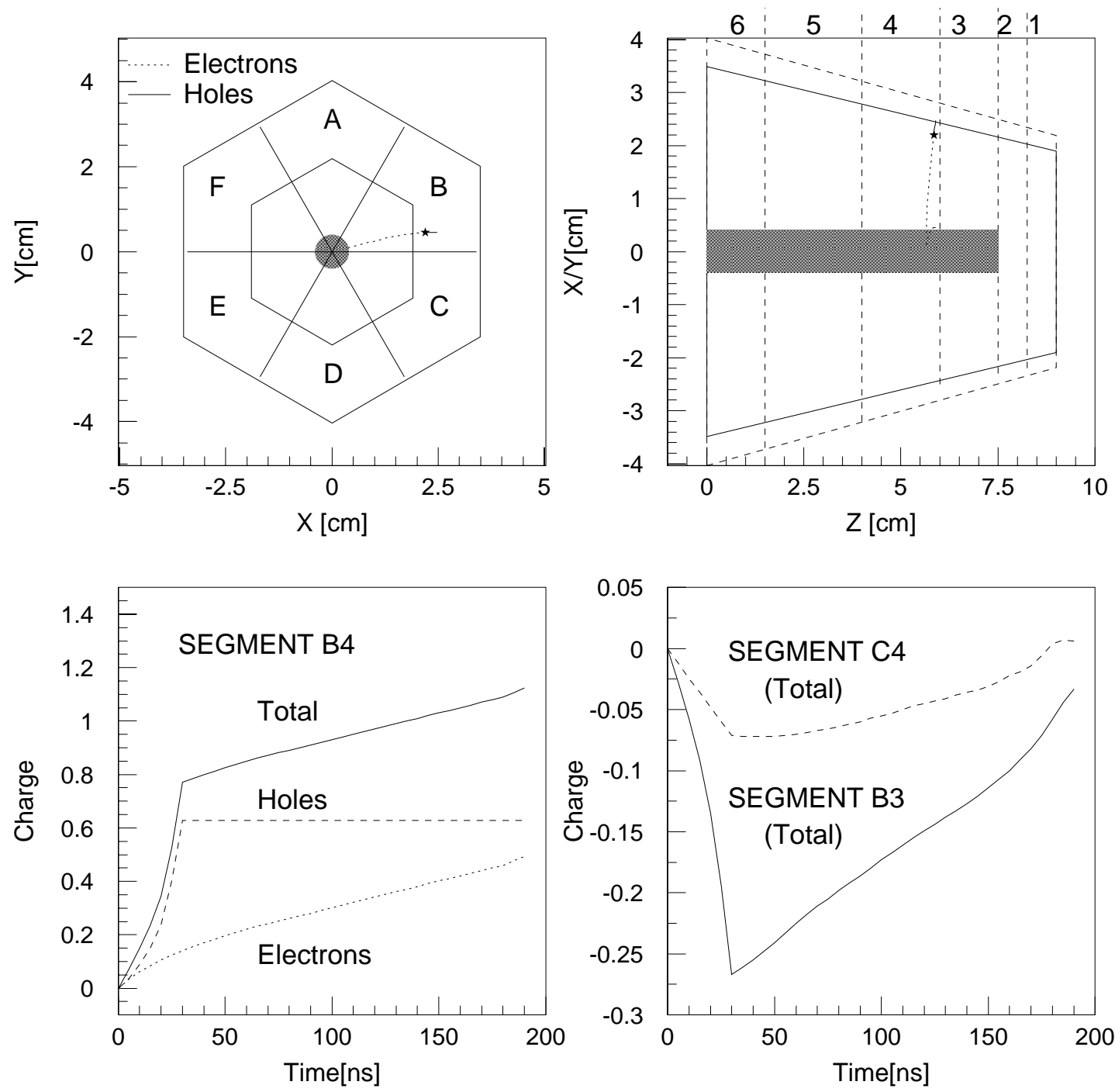

Fig. 3. The upper part shows the calculated path of electrons and holes in the GRETA prototype detector. The geometry as well as the arrangement of segment boundaries is shown. The interaction at $\mathrm{x}=22 \mathrm{~mm}, \mathrm{y}=4.5 \mathrm{~mm}$ and $\mathrm{z}=58.5 \mathrm{~mm}$ is marked with a star in segment B4. Calculated signals for B4 and the nearest neighbors B3 and $\mathrm{C} 4$ are plotted in the lower part. 


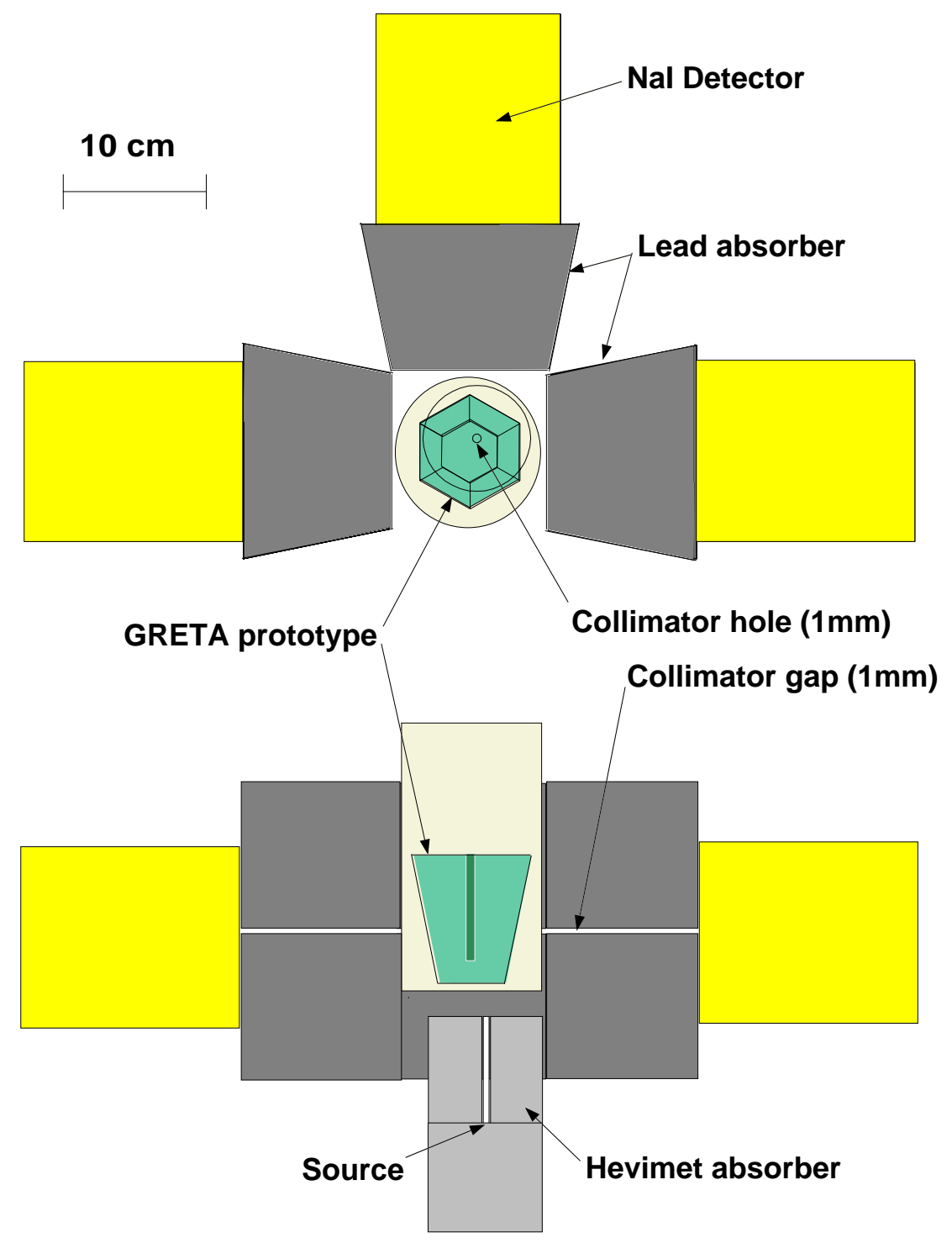

Fig. 4. Experimental setup used for the measurements. The upper figure shows a top view on the coincidence setup and the lower part illustrates a side view of the vertical arrangement of collimation and detector systems. 

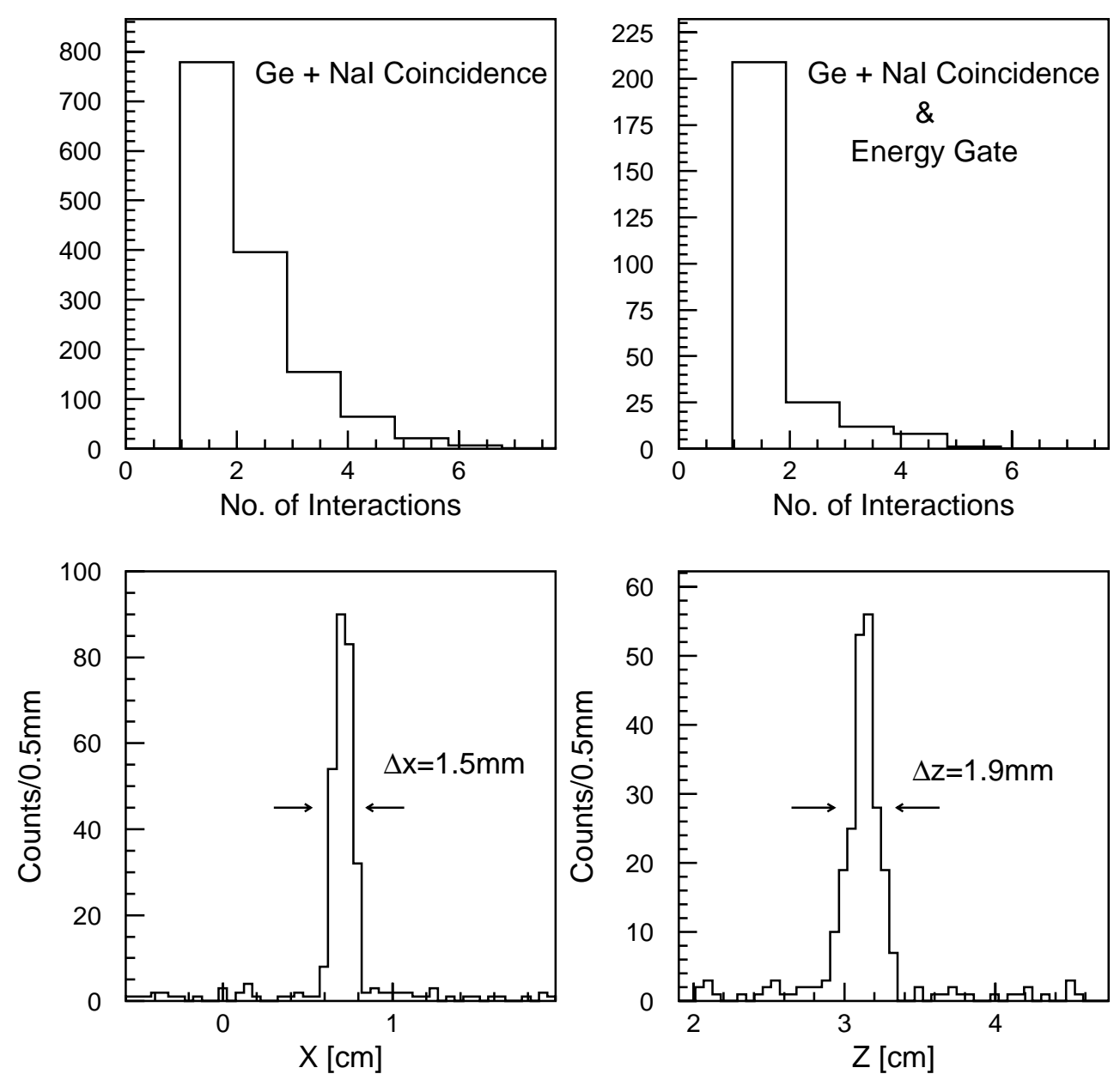

Fig. 5. Calculated number of interactions and position distribution. The multiplicity distributions show the effect of requiring the energy for a 90 degree Compton scattering on the number of interactions in the GRETA prototype detector. $\Delta x$ represents the radial uncertainty, $\Delta \mathrm{z}$ the width of the distribution of interactions in the depth. 


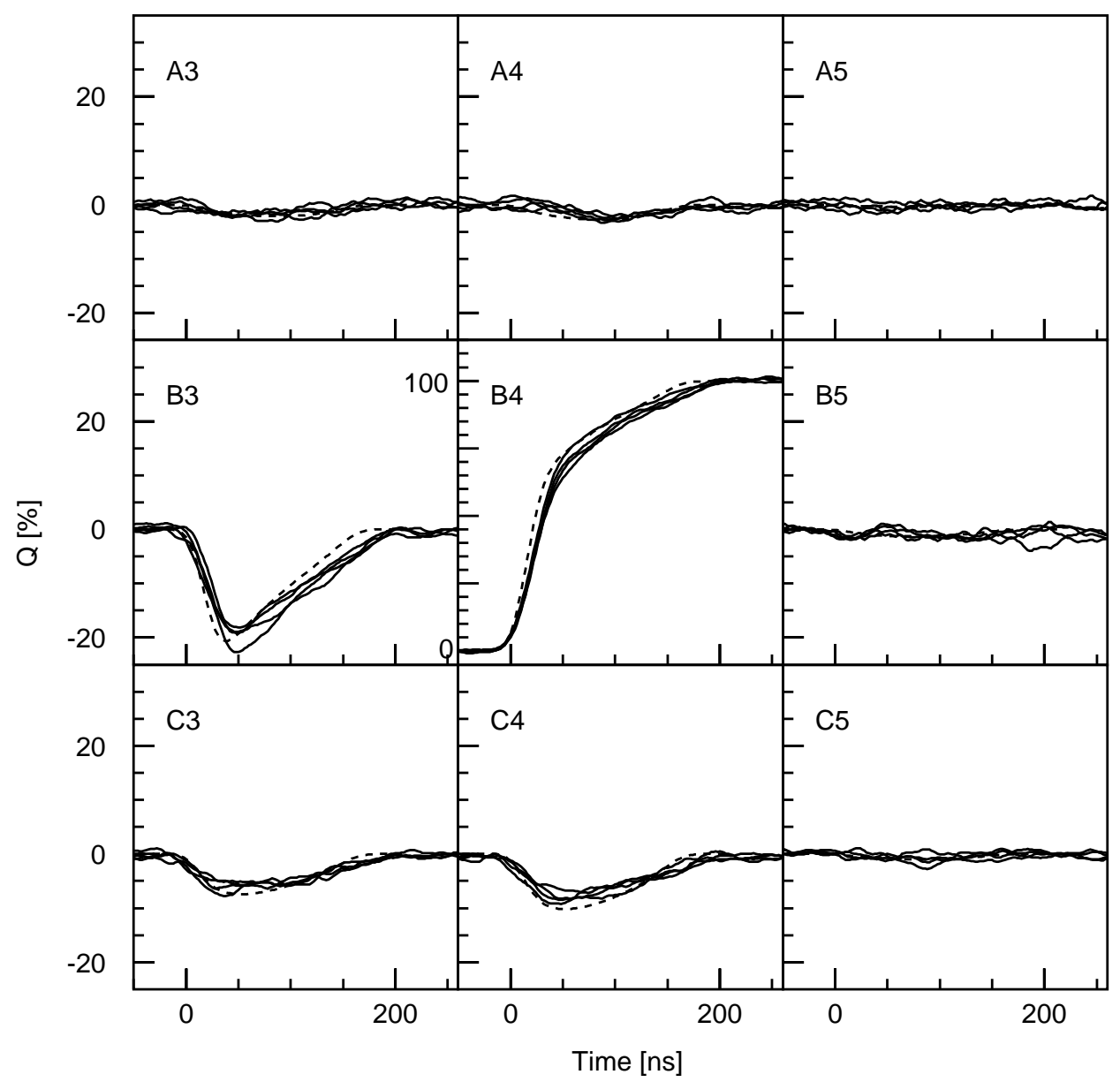

Fig. 6. Measured set of segment signals at $\mathrm{x}=22 \mathrm{~mm}, \mathrm{y}=4.5 \mathrm{~mm}$ and $\mathrm{z}=58.5 \mathrm{~mm}$ $(\Delta \mathrm{z}=1.5 \mathrm{~mm})$. Segment B4 contains the interaction which is assumed to be $4.5 \mathrm{~mm}$ away from segment $\mathrm{C} 4$ and $1.5 \mathrm{~mm}$ away from segment B3. The dashed line indicates the calculated signals for this location. The calculated signals are the same as in fig. 3 . 


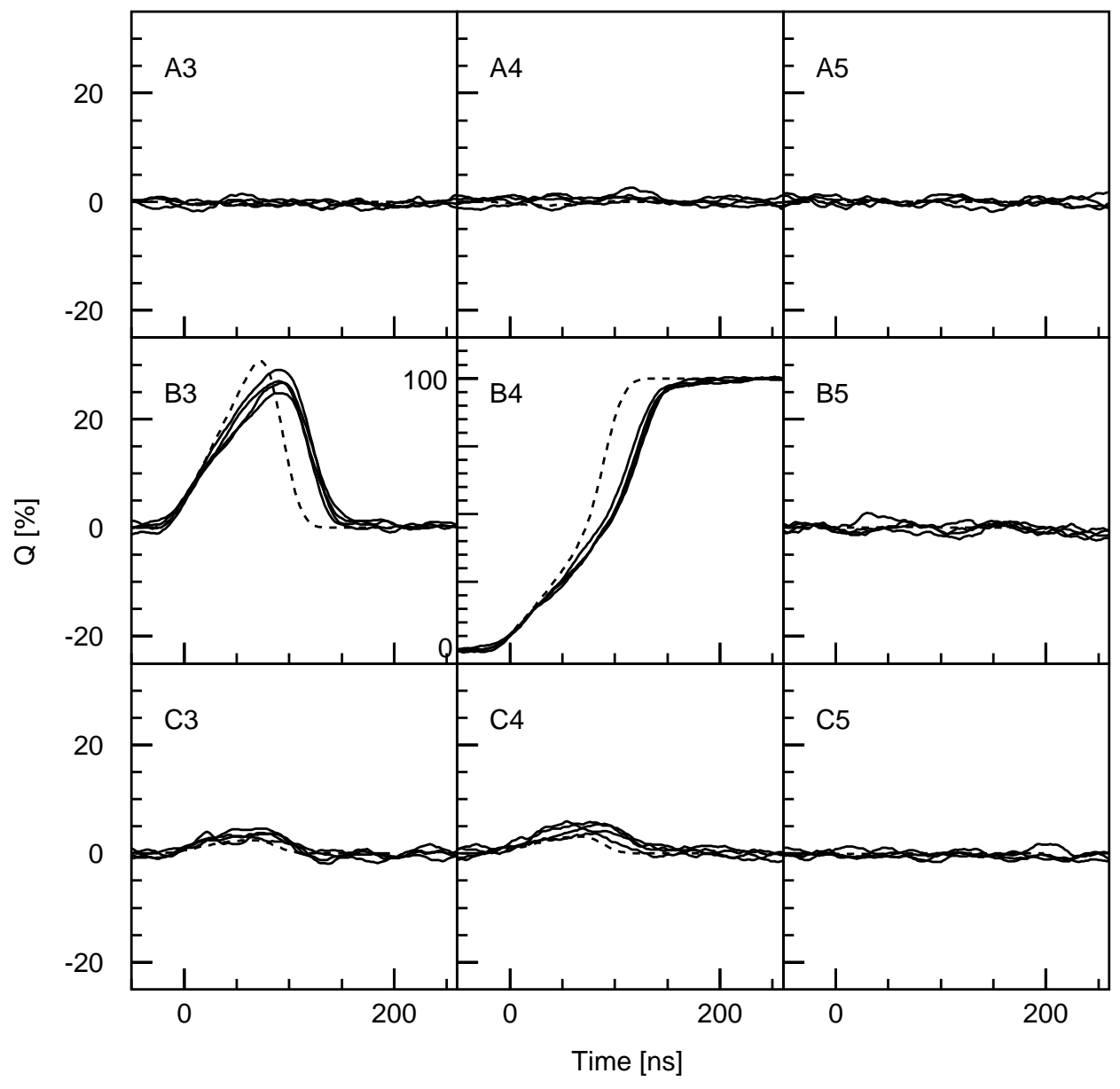

Fig. 7. Same as 6 except the location is at $\mathrm{x}=14 \mathrm{~mm}, \mathrm{y}=4.5 \mathrm{~mm}$ and $\mathrm{z}=58.5 \mathrm{~mm}$ $(\Delta \mathrm{z}=1.5 \mathrm{~mm})$. 


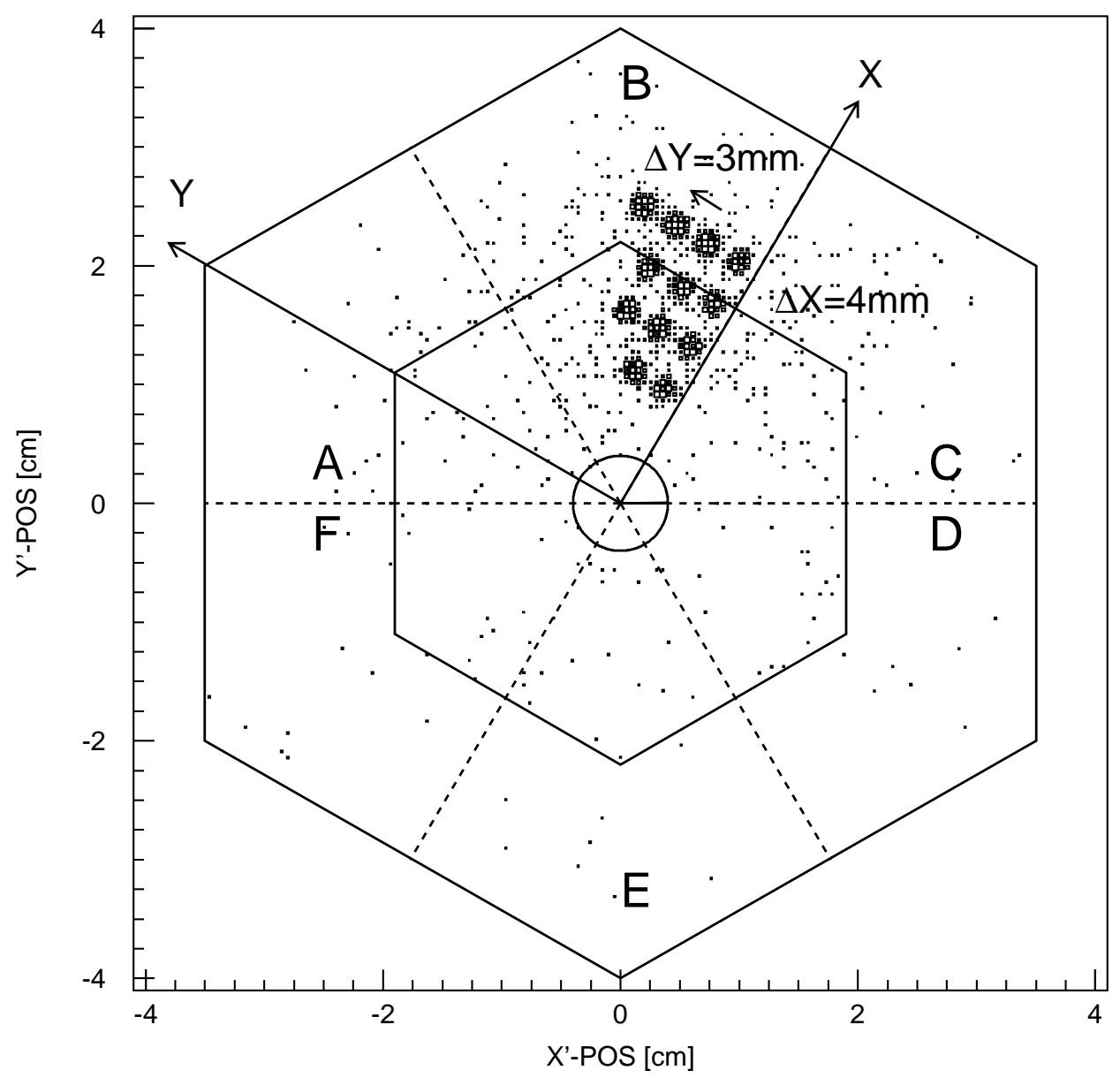

Fig. 8. Calculated positions of interactions for the 12 different collimator locations per z-layer. The dashed line indicates the segment boundaries. 


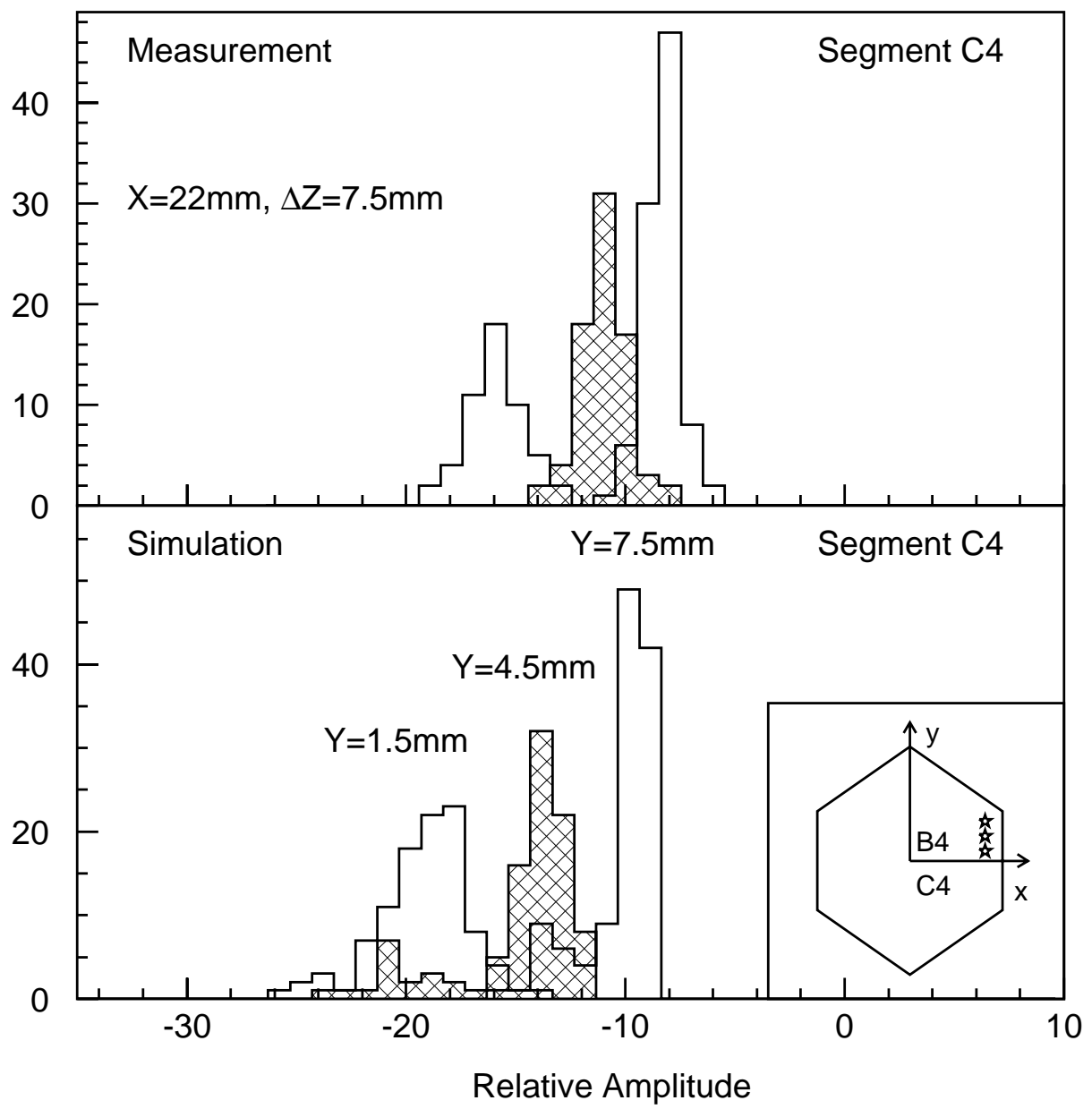

Fig. 9. Measured (top) and calculated (bottom) distributions of maximum charge amplitudes of transient induced signals in segment C4 of the GRETA prototype detector. The distribution in the center is hatched to distinguish the three different y positions perpendicular to the B4/C4 boundary. The insert illustrates the relative positions. 


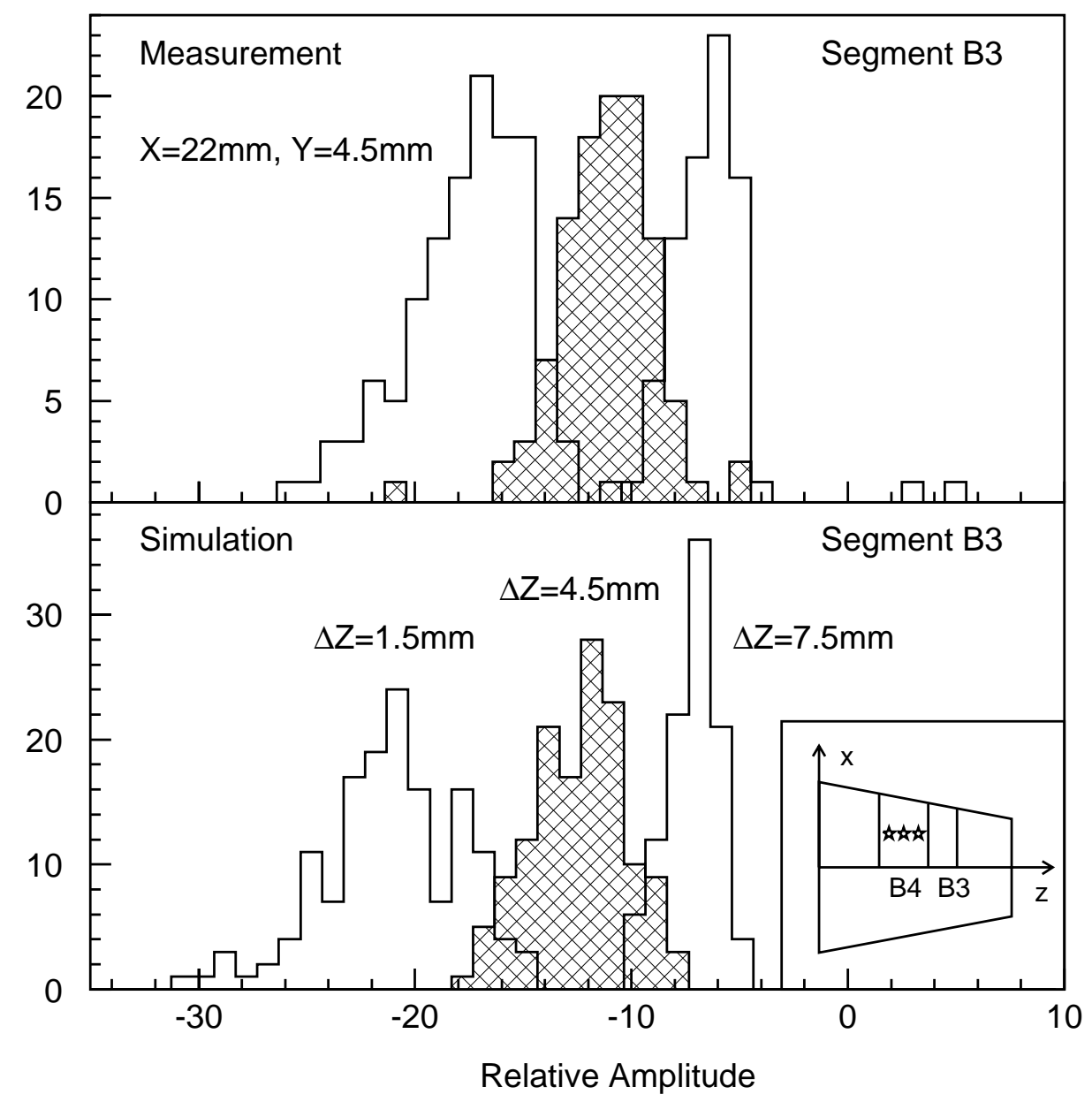

Fig. 10. As fig. 9, except that transient signal amplitudes were obtained in segment B3 for different distances along z perpendicular to the B3/B4 boundary. 


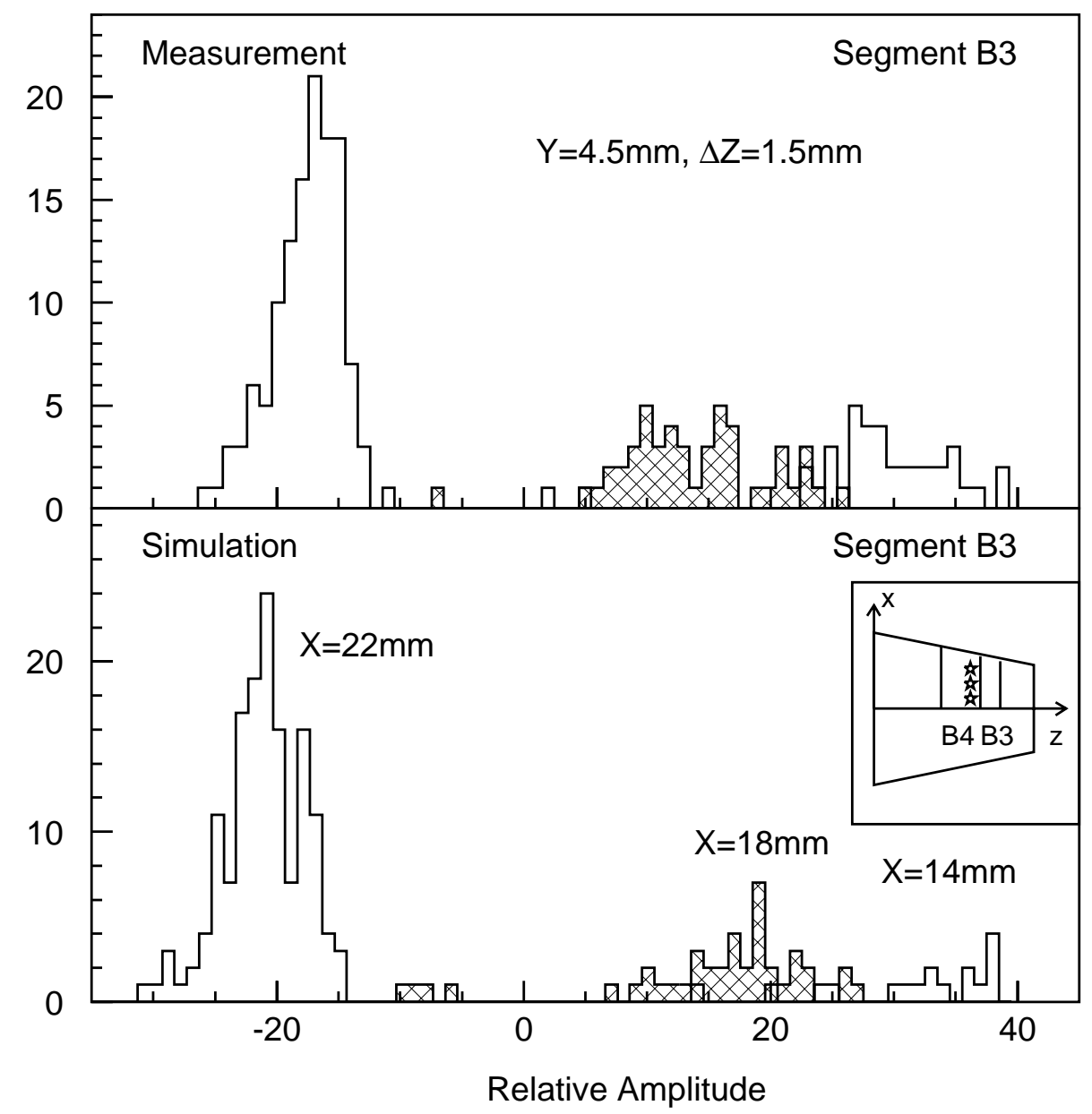

Fig. 11. As fig. 10, except that transient signal amplitudes in segment B3 are plotted for different $\mathrm{x}$-values at fixed $\mathrm{y}$ and $\mathrm{z}$-values. 

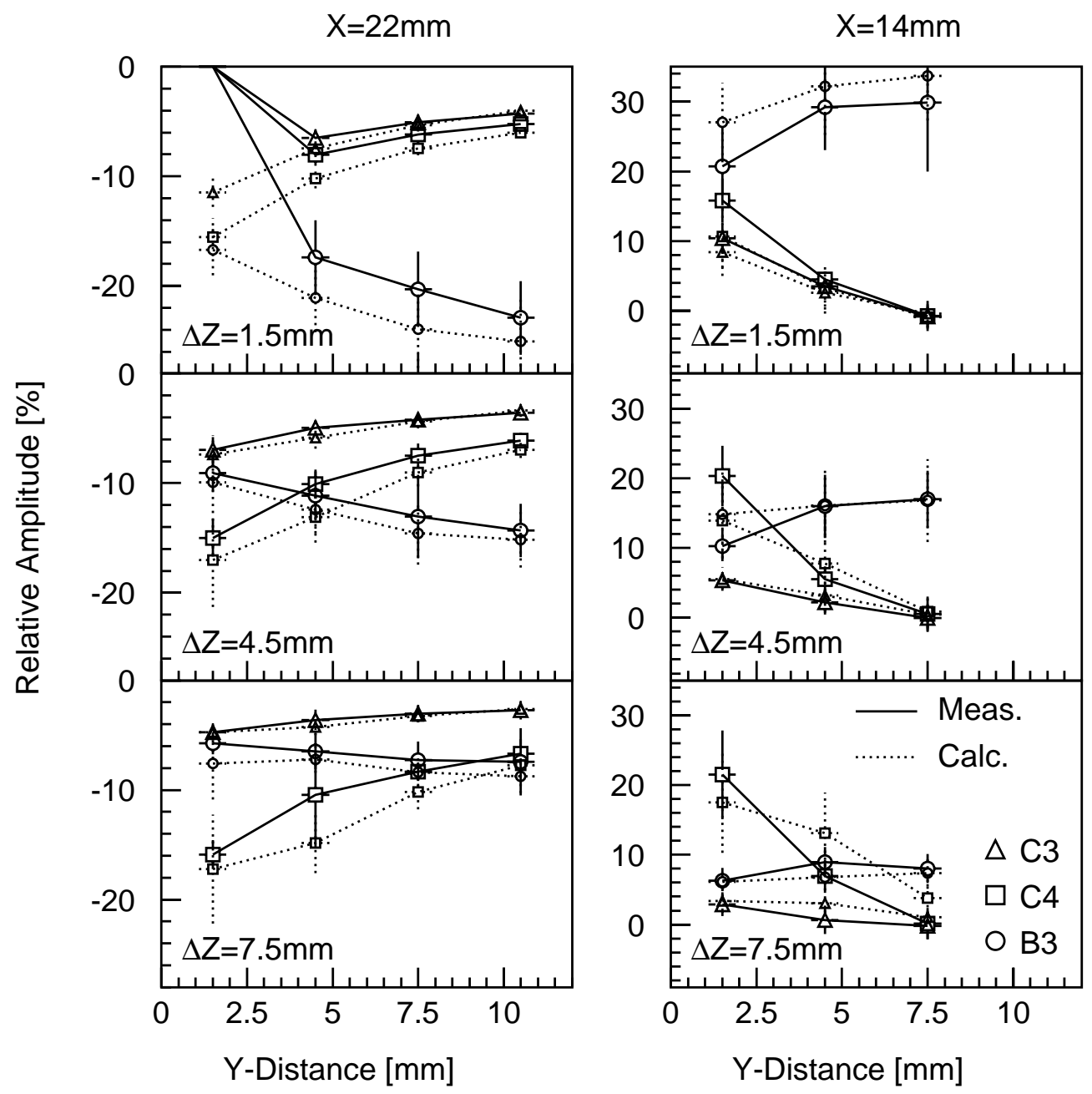

Fig. 12. Measured (solid lines) and calculated (dotted lines) mean and width values of maximum transient signal charge amplitudes for segment C3 (triangle), C4 (square) and B3 (circle). 


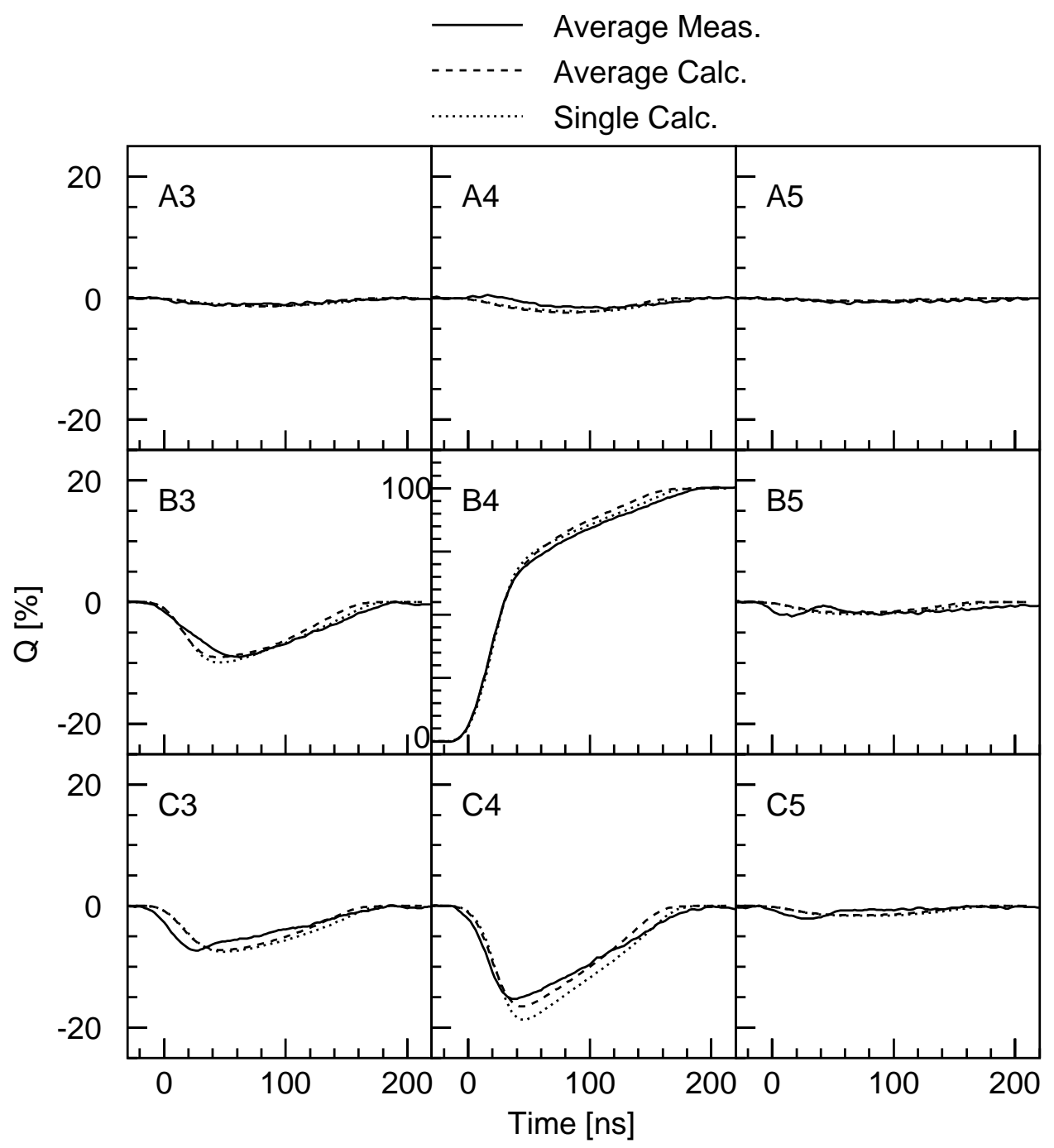

Fig. 13. Measured set of averaged segment signals at $\mathrm{x}=22 \mathrm{~mm}, \mathrm{y}=1.5 \mathrm{~mm}$ and $\mathrm{z}=55.5 \mathrm{~mm}(\Delta \mathrm{z}=4.5 \mathrm{~mm})$ close to the edge of the crystal. Segment B4 contains the interaction resulting in the net charge signal. For comparison to the measured charge signals (solid lines) averaged calculated signals (dashed lines) and calculated signals based on single interactions at the targeted location (dotted lines) are shown. 


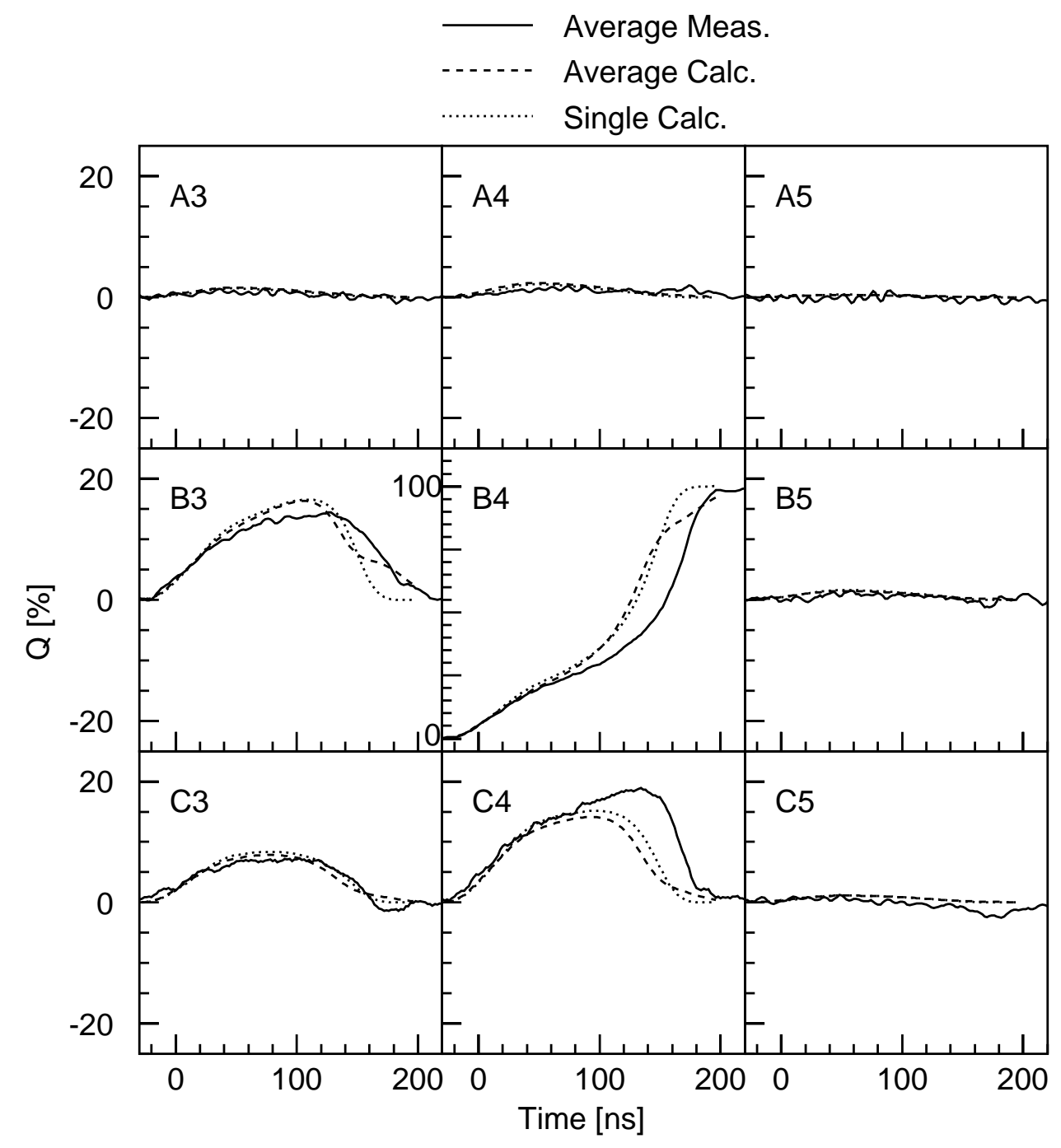

Fig. 14. Same as fig. 13 but for a different location at $\mathrm{x}=10 \mathrm{~mm}, \mathrm{y}=1.5 \mathrm{~mm}$ and $\mathrm{z}=55.5 \mathrm{~mm}(\Delta \mathrm{z}=4.5 \mathrm{~mm})$, close to the inner hole of the crystal. 


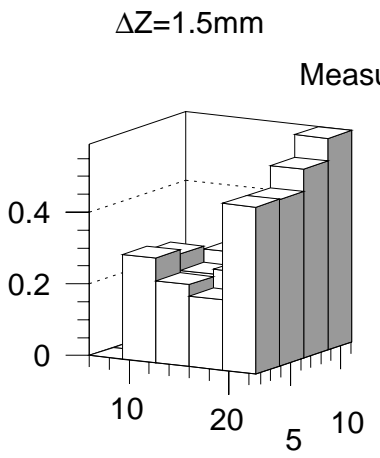

$\Delta \mathrm{Z}=4.5 \mathrm{~mm}$

Measured Sensitivities (Averaged Signals)
$\Delta \mathrm{Z}=7.5 \mathrm{~mm}$
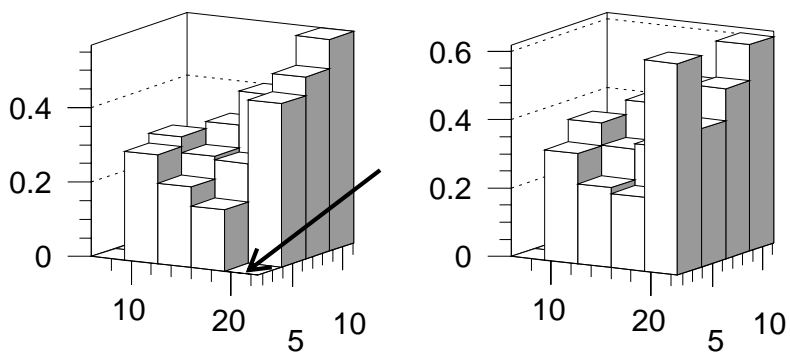

Calculated Sensitivities (Averaged Signals)
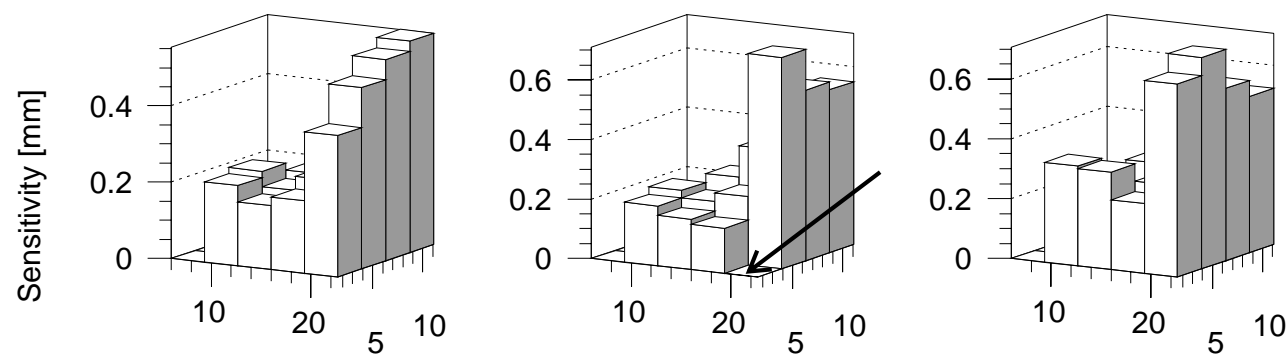

Calculated Sensitivities (Single Signals)
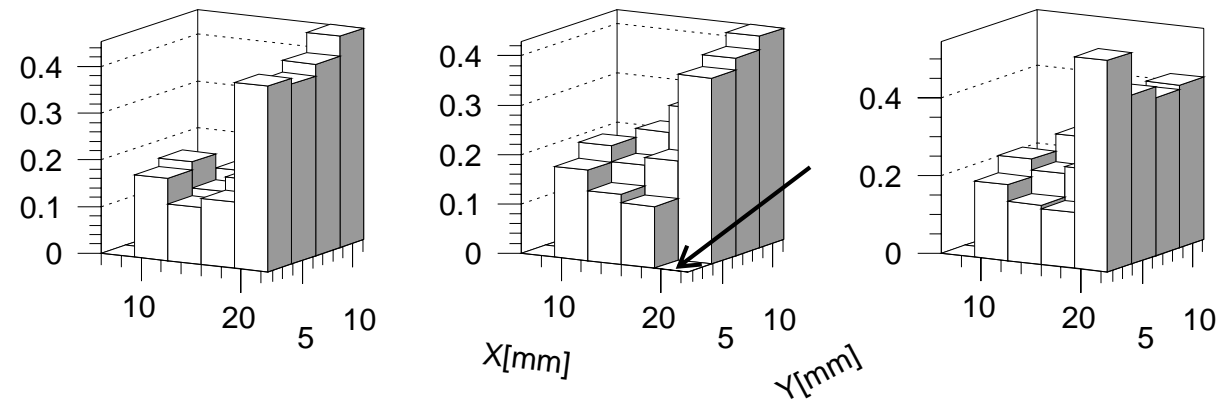

Fig. 15. Sensitivities obtained for the location at $\mathrm{x}=22 \mathrm{~mm}, \mathrm{y}=1.5 \mathrm{~mm}$ and $\mathrm{z}=55.5 \mathrm{~mm}$, marked by the arrow. The upper part shows measured and the lower two rows show calculated sensitivities. The two upper parts are determined after averaging the signals for one location while the lower part is calculated based on a single interaction at the aimed location. 


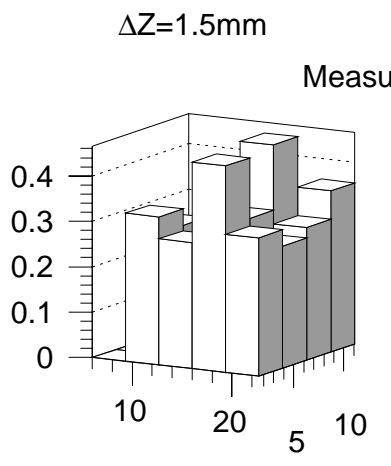

$\Delta \mathrm{Z}=4.5 \mathrm{~mm}$

$\Delta \mathrm{Z}=7.5 \mathrm{~mm}$

leasured Sensitivities (Averaged Signals)

Calculated Sensitivities (Averaged Signals)
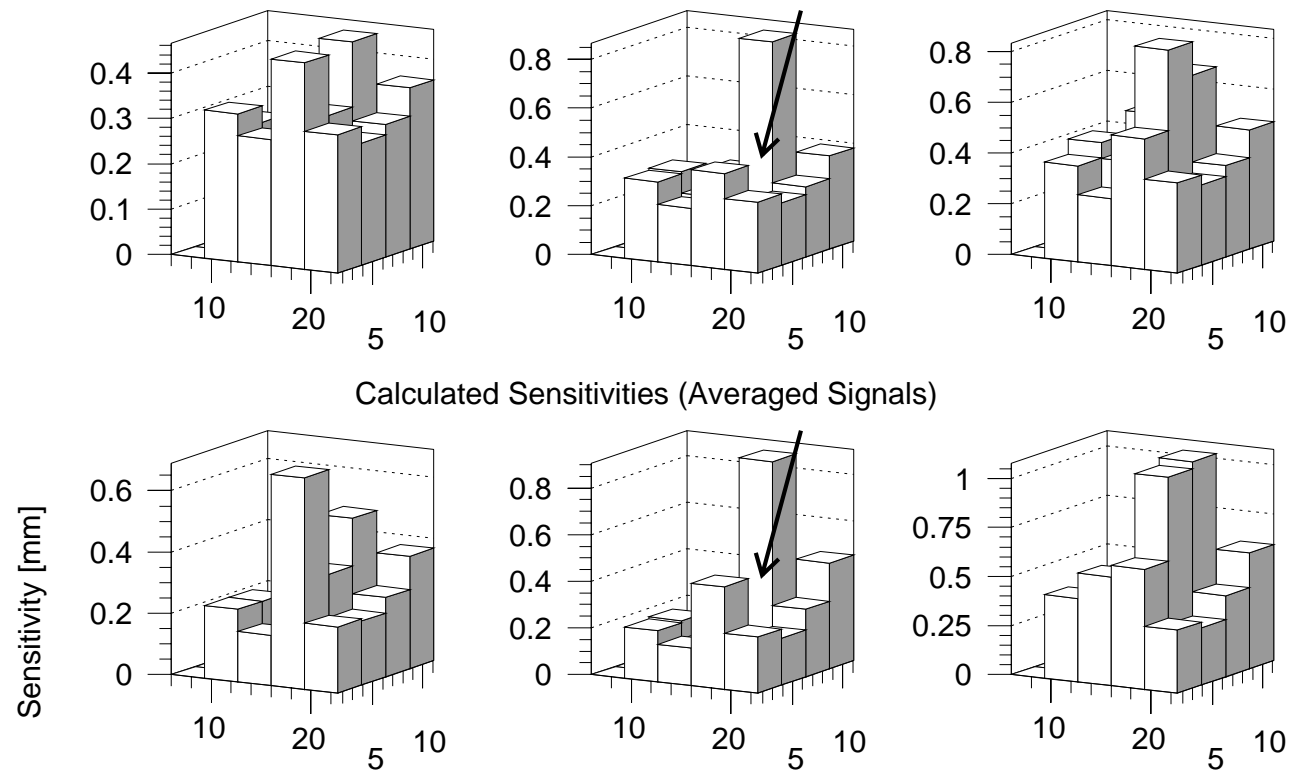

Calculated Sensitivities (Single Signals)
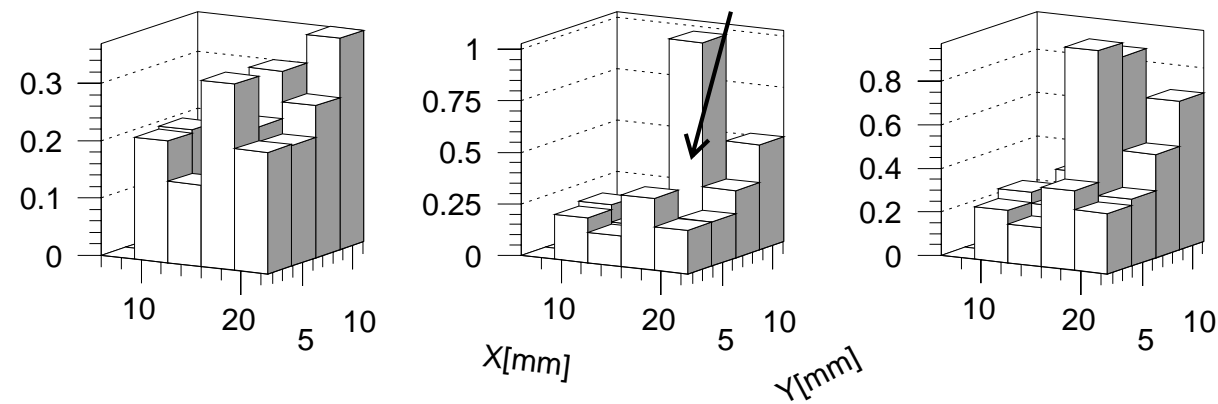

Fig. 16. Sensitivities obtained for the location at $\mathrm{x}=18 \mathrm{~mm}, \mathrm{y}=4.5 \mathrm{~mm}$ and $\mathrm{z}=55.5 \mathrm{~mm}$, marked by the arrow, as in fig. 15 . These $\mathrm{x}$ and $\mathrm{y}$ positions represent the lowest sensitivity obtained for all measured combinations of locations. 


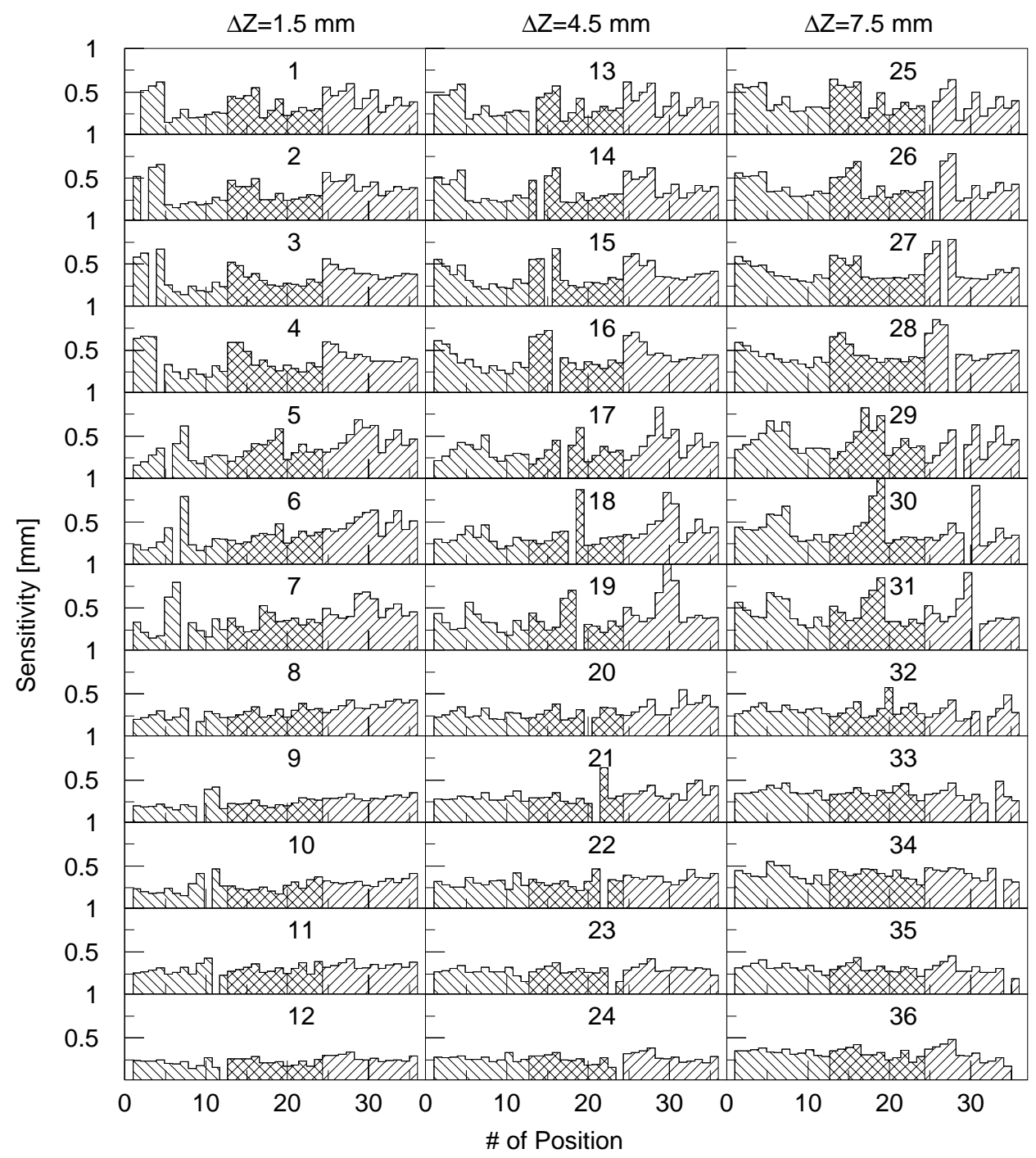

Fig. 17. Measured sensitivity for all combinations of positions. In total, 36 locations have been measured in three different $\mathrm{z}$ layers. The three different columns as well as the three different hatch styles indicate the three layers in the depth of the crystal. 


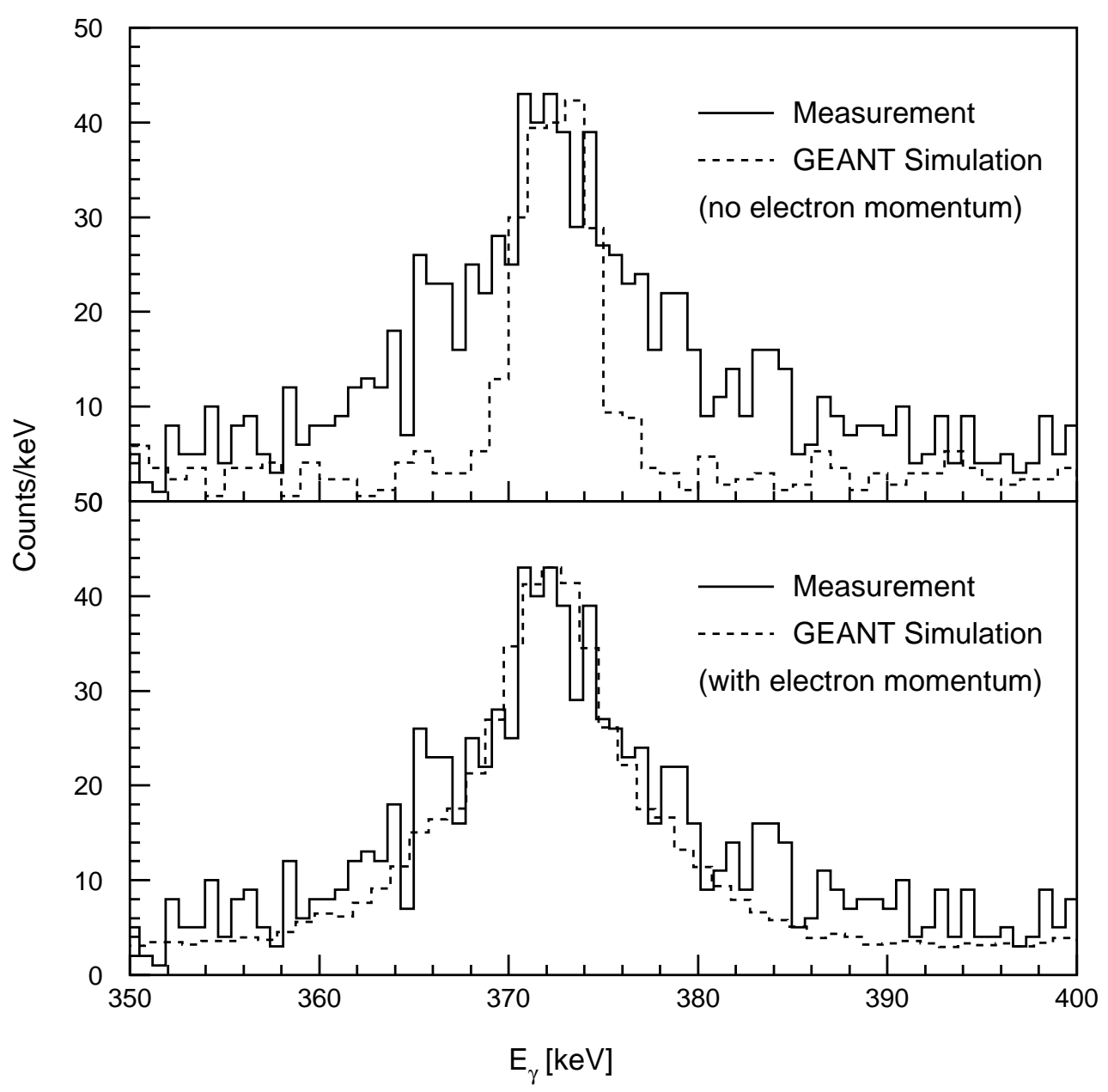

Fig. 18. Partial energy spectra measured in segment B4 of the GRETA prototype detector. The peak at $374 \mathrm{keV}$ reflects the requirement of a coincidence between the Ge and one of the NaI detectors. The dashed lines show calculated spectra assuming the same geometry as in the experiment. 